\title{
Microbial and Enzymatic Degradation of Synthetic Plastics
}

\author{
Nisha Mohanan', Zahra Montazer², Parveen K. Sharma' and David B. Levin ${ }^{1 *}$ \\ ${ }^{1}$ Department of Biosystems Engineering, University of Manitoba, Winnipeg, MB, Canada, ${ }^{2}$ Faculty of Food Engineering, \\ The Educational Complex of Agriculture and Animal Science, Torbat-e-jam, Iran
}

Synthetic plastics are pivotal in our current lifestyle and therefore, its accumulation is a major concern for environment and human health. Petroleum-derived (petro-)polymers such as polyethylene (PE), polyethylene terephthalate (PET), polyurethane (PU), polystyrene (PS), polypropylene (PP), and polyvinyl chloride (PVC) are extremely recalcitrant to natural biodegradation pathways. Some microorganisms with the ability to degrade petro-polymers under in vitro conditions have been isolated and characterized. In some cases, the enzymes expressed by these microbes have been cloned and sequenced. The rate of polymer biodegradation depends on several factors including chemical structures, molecular weights, and degrees of crystallinity. Polymers are large molecules having both regular crystals (crystalline region) and irregular groups (amorphous region), where the latter provides polymers with flexibility. Highly crystalline polymers like polyethylene (95\%), are rigid with a low capacity to resist impacts. PET-

OPEN ACCESS

Edited by:

Sanket J. Joshi,

Sultan Qaboos University, Oman

Reviewed by:

Shashi Kant Bhatia,

Konkuk University, South Korea Wei-Min Wu,

Stanford University, United States

*Correspondence:

David B. Levin

david.levin@umanitoba.ca

Specialty section:

This article was submitted to

Microbiotechnology,

a section of the journal

Frontiers in Microbiology

Received: 06 July 2020

Accepted: 20 October 2020

Published: 26 November 2020

Citation:

Mohanan N, Montazer Z, Sharma PK and Levin DB (2020) Microbial and Enzymatic Degradation of Synthetic Plastics.

Front. Microbiol. 11:580709. doi: 10.3389/fmicb.2020.580709 based plastics possess a high degree of crystallinity (30-50\%), which is one of the principal reasons for their low rate of microbial degradation, which is projected to take more than 50 years for complete degraded in the natural environment, and hundreds of years if discarded into the oceans, due to their lower temperature and oxygen availability. The enzymatic degradation occurs in two stages: adsorption of enzymes on the polymer surface, followed by hydro-peroxidation/hydrolysis of the bonds. The sources of plasticdegrading enzymes can be found in microorganisms from various environments as well as digestive intestine of some invertebrates. Microbial and enzymatic degradation of waste petro-plastics is a promising strategy for depolymerization of waste petroplastics into polymer monomers for recycling, or to covert waste plastics into higher value bioproducts, such as biodegradable polymers via mineralization. The objective of this review is to outline the advances made in the microbial degradation of synthetic plastics and, overview the enzymes involved in biodegradation.

Keywords: synthetic polymers, polyethylene, polyethylene terephthalate, polyurethane, polystyrene, polypropylene, polyvinyl chloride, cutinase

\section{INTRODUCTION}

Petroleum-derived (petro-)plastics have many desirable characteristics. They are lightweight and have very stable chemical and physical properties, which makes them highly durable. Production methods are well established and very high capacity, resulting in very low cost. Consequently, they have become ubiquitous in the global economy (Barnes et al., 2009; Andrady, 2011; Hidalgo-Ruz et al., 2012). However, accumulation of petro-plastics wastes in the environment is now a major 
global problem because these materials are recalcitrant to natural biodegradation processes. As a result of their massive accumulation in municipal waste systems, micro- and nano-sized plastic particles are now ubiquitous in both terrestrial and aquatic ecosystems (Álvarez-Hernández et al., 2019; Bergmann et al., 2019). About 335 million tons of plastics were manufactured worldwide in 2016 (Plastics Europe, 2017). Synthetic plastics such as polyethylene terephthalate (PET), polyethylene (PE), polyurethane (PUR), polystyrene (PS), polypropylene (PP), and polyvinyl chloride (PVC) have been extensively utilized in a widerange of industrial and domestic applications (Shah et al., 2008).

Environmental pollution by plastic waste was first reported in the 1970s (Carpenter and Smith, 1972). The growing amount of plastic waste has become a global concern. Despite increasing efforts to reduce the plastic waste by disposing off through segregated collection and recycling, a sizeable amount of plastic solid waste is still landfilled. From whole plastic production by 2017 (8,300 million tons), after recycling, incineration (energy recovery) of wastes and calculating in-use plastics in domestic; around $60 \%$ have been left in the environment including 95\% in landfills and 5\% in the oceans and other terrestrial areas (Ragaert et al., 2017). Plastic debris in the environment is degraded in nature by photo-, bio-, and thermo-oxidative depolymerization as well as friction (Barnes et al., 2009; Browne et al., 2011). Although biodegradation of these plastics is feasible in the natural environment, it can take long periods of time: from 50 to more than 100 years (Table $\mathbf{1}$ ).

Based on the degradation pathways, these synthetic plastics have been divided into two groups, plastics with a carboncarbon backbone and plastics with heteroatoms in the main chain. PE, PS, PP, and PVC plastics have a backbone which is only built of carbon atoms (Figure 1). PET and PU have heteroatoms in the main chain (Figure 1). Several biodegradable aliphatic polyesters such as polyhydroxyalkanoate (PHA) and polylactic acid (PLA), were produced and may be used as alternatives to some petro-plastics (Tokiwa et al., 2009; Bhatia et al., 2019a,b). Polyhydroxyalkanoates (PHAs) have emerged as a sustainable choice due to their putative high biodegradability in different environments, biocompatibility, chemical diversity, their manufacture from renewable carbon resources, and release of non-polluting and non-toxic products after degradation (Bhatia et al., 2019a,b). PHAs are synthesized and accumulated by many prokaryotic microorganisms as

TABLE 1 | Selected properties of major synthetic thermoplastic polymers (Ojeda, 2013).

\begin{tabular}{lccc}
\hline Polymer & Density (g/L) & Crystallinity (\%) & Life span (years) \\
\hline PET & 1.35 & $0-50$ & 450 \\
LDPE & $0.91-0.93$ & 50 & $10-600$ \\
HDPE & $0.94-0.97$ & 70 & $>600$ \\
PS & $1.03-1.09$ & 0 & $50-80$ \\
PP & $0.90-0.91$ & 50 & $10-600$ \\
PVC & $1.35-1.45$ & 0 & $50-150$
\end{tabular}

PET, Polyethylene terephthalate; LDPE, Low density polyethylene; HDPE, High density polyethylene; PS, Polystyrene; PP, Polypropylene; PVC, Polyvinyl chloride. storage compounds for carbon and energy when a major noncarboneous nutrient (e.g., nitrogen or phosphorus) is limiting. The accumulation of these polymers facilitates enhanced survival under environmental stress conditions. However, the most commonly used plastics are still synthetic polymers derived from petrochemical hydrocarbons (Geyer et al., 2017).

Degradation of plastics by microbial and/or enzymatic means (Figure 2) is a promising strategy to depolymerize waste petro-plastics into monomers for recycling, or mineralize them into carbon dioxide, water, and new biomass, with concomitant production of higher-value bioproducts (Grima et al., 2000; Montazer et al., 2019, 2020a). Biodegradation of plastics involves excretion of extracellular enzymes by the microorganism, attachment of enzyme to the surface of plastic, hydrolysis to short polymer intermediates, which are ultimately assimilated by microbial cells as carbon source to release $\mathrm{CO}_{2}$. Despite the fact that these plastics represent non-natural chemicals, several microorganisms capable of metabolizing these polymers have been identified in recent years. Over 90 microorganisms, including bacteria and fungi, have been known to degrade petroleum-based plastics (Jumaah, 2017) mostly in vitro condition.

Biodegradation is a complex process which is dependent on several factors, such as availability of a substrate, surface characteristics, morphology, molecular weight of the polymers (Figure 3) and therefore, an exact definition of biodegradation is lacking (Albertsson et al., 1987; Ammala et al., 2011; Harrison et al., 2018). Further, biodegradation has been measured by a wide-range of variables, including substrate weight loss, changes in the mechanical properties and/or the chemical structure of the polymer and the percentage of carbon dioxide emission. Early microbial biodegradation experiments attempted to demonstrate that microbial activity could result in changes in the physical characteristics of plastics, such as tensile strength, crystallinity, and water uptake (Pirt, 1980; Albertsson and Karlsson, 1990). The identification and genetic engineering of these plastic-degrading microorganisms and/or enzymes will provide an opportunity to improve plastic recycling and thereby reduce environmental plastic pollution by means of assimilation of plastic waste into carbon source or degradation of plastics waste into valuable alkane products via microbial biotechnology. The biodegradation mechanisms of petro-plastics are likely related to the types of bonds in the polymeric chains (since the active sites of related enzymes are individual for any specific bond). Thus, mechanisms of petro-plastic degradation can be classified into three groups: (i) Polymers with carbon back-bones; (ii) Polymers with ester-bond back-bones and side-chains; and (iii) Polymers with hetero/carbamate(urethane) bonds.

The present review outlines the recent advances made in the microbial degradation of synthetic plastics like polyethylene PE, polystyrene, polypropylene, polyvinyl chloride, PET, and PU and, overview the enzymes involved in biodegradation. Therefore, the study contributes to the existing knowledge in the field of microbial and/or enzymatic degradation of the synthetic plastics. Polymer degradation by microbial and enzymatic means is a promising strategy to convert plastic waste into carbon dioxide, polymer monomers, and possibly value-added compounds. 
Polyethylene terephthalate (PET)<smiles>CCCOC(=O)c1ccc(C(=O)OC)cc1</smiles><smiles>CCOCCOC(=O)Nc1ccc(Cc2ccc(NC(C)=O)cc2)cc1</smiles>

\section{Polyurethane (PU)}

\section{$n$}

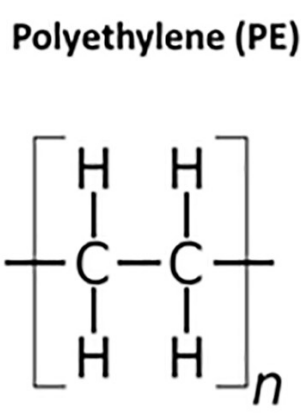

Polyvinyl chloride (PVC)

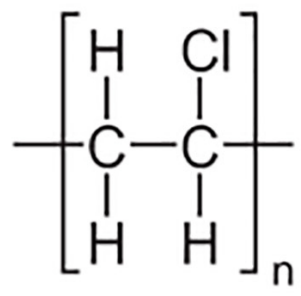

Polypropylene (PP)

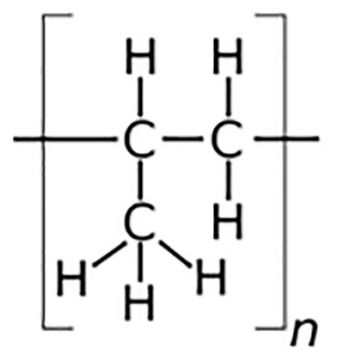

Polystyrene (PS)

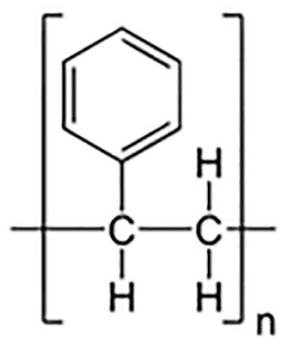

FIGURE 1 | Structures of major commercial synthetic polymers.

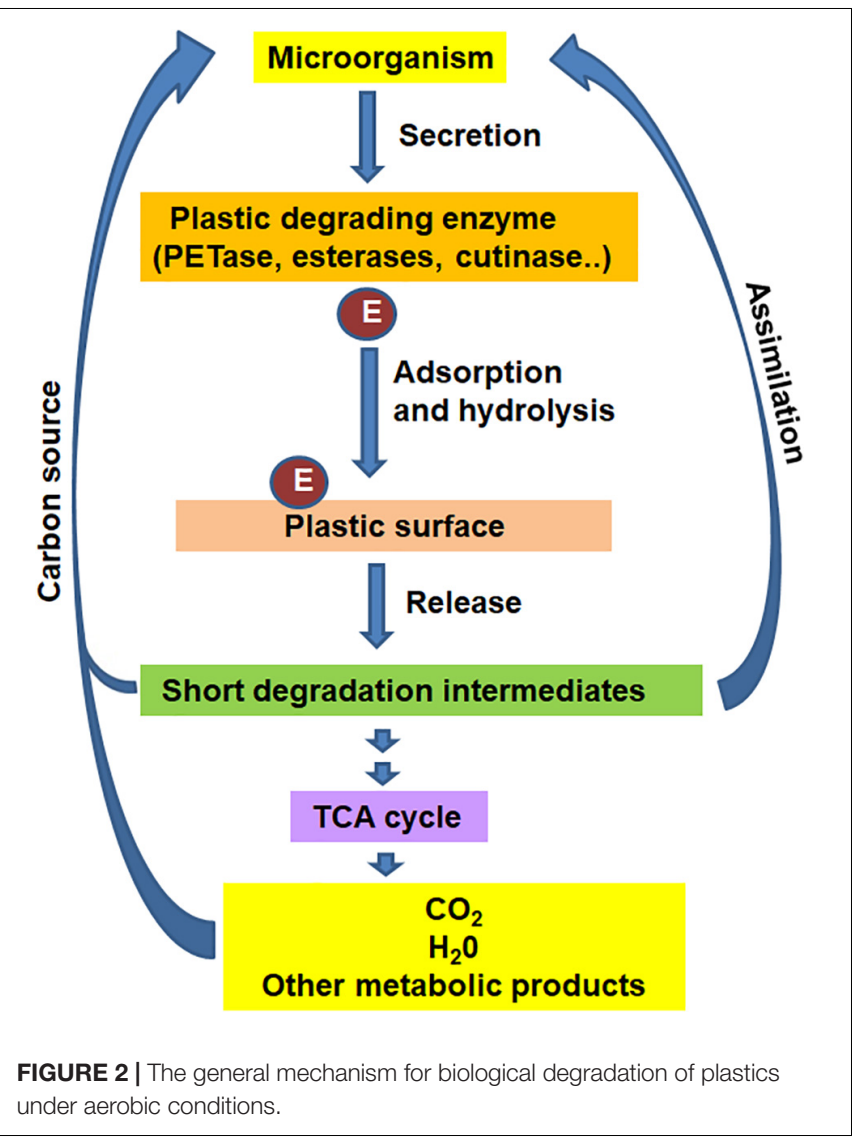

\section{PETRO-PLASTICS WITH CARBON BACK-BONES}

Polyethylene, polypropylene, polystyrene, and polyvinyl chloride have a backbone which is solely made of carbon atoms. These polymers constitute the main packaging materials (Plastics Europe, 2017). UV-radiation and oxygen are the major initiation factors responsible for degradation of polymers with a carbon-carbon backbone, leading to fragmentation or chain scission. Photo-initiated oxidative degradation of PE, PP, and PS leads to reduced molecular weight and formation of carboxylic end groups, and UV-light initiates dechlorination in PVC polymers. The resulting smaller polymer fragments are susceptible to biodegradation and, abiotic degradation precedes biodegradation. Plastics Additives (1998) demonstrated that only $\sim 0.1 \%$ per year of the carbon that makes up the polymer is converted into $\mathrm{CO}_{2}$ by biodegradation under optimum laboratory conditions.

\section{Polyethylene}

\section{PE Degrading Microorganisms}

$\mathrm{PE}$ is composed of a linear chain of carbons held together by hydrogen bonds (Figure 1). PE usually has a semi-crystalline structure and is extremely resistant to biodegradation. Based on different manufacturing processes and subsequently different arrangements of the linear chains, PE polymers can have different densities and 3-dimensional and physical structures, low molecular weight polyethylene (LMWPE), linear low-density 


\section{Biodegradation controlling factors}

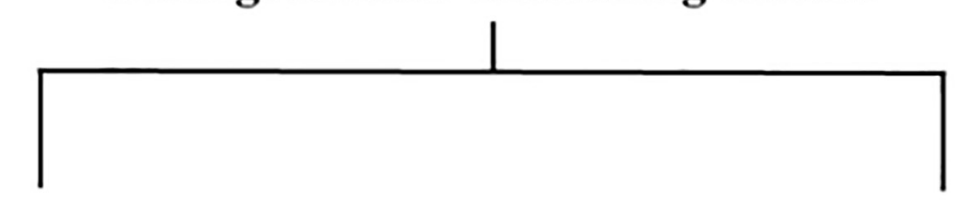

\section{Exposure conditions}

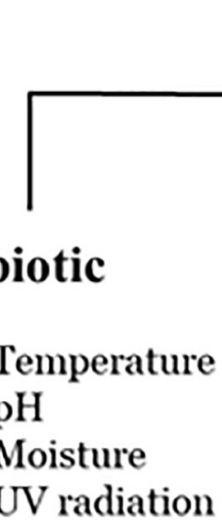

\section{Polymer properties}

- Molecular weight

- Size and shape

- Crystallinity

- Functional groups

- Cross-linking

- Blend

- Copolymers

- Additives
- $\mathrm{pH}$

- Moisture

- UV radiation
- Hydrophobicity

- Extracellular enzymes

- Nutrients

- Biosurfactants

FIGURE 3 | Factors affecting polymer biodegradation (adapted from Kijchavengkul and Auras, 2008).

polyethylene (LLDPE), low-density polyethylene (LDPE), and high-density polyethylene (HDPE).

Biodegradation of the PE plastic wastes by microbial isolates has been a major topic of research (Nowak et al., 2011; Kyaw et al., 2012; Gajendiran et al., 2016; Sen and Raut, 2016). Microorganisms capable of hydrolyzing PE have been isolated from soil, sea water, compost and activated sludge (Montazer et al., 2020a). Bacteria in the gut of the greater wax worm, Galleria melonella have been found capable of hydrolyzing polyethylene (PE) (Yang et al., 2014; Bombelli et al., 2017; Cassone et al., 2020). Besides PET, biotechnological conversion of PE through pyrolysis and transformation or direct transformation of $\mathrm{PE}$ to polyhydroxyalkanoate in cells, has been reported in different Pseudomonads (Ward et al., 2006; Kenny et al., 2008; Guzik et al., 2014; Montazer et al., 2019).

Bacterial species, such as Bacillus spp. (Sudhakar et al., 2008; Abrusci et al., 2013), Rhodococcus spp. (Bonhomme et al., 2003; Gilan et al., 2004; Fontanella et al., 2010), and Pseudomonas spp. (Rajandas et al., 2012), and fungi, such as Aspergillus and Fusarium (Hasan et al., 2007; Sahebnazar et al., 2010), were shown to depolymerize PE after some forms of pretreatment such as Ultraviolet (UV) and/or thermal treatments, which render the carbon chains of polymer sensitive to biodegradation (Ammala et al., 2011). Microbial degradation of untreated $\mathrm{PE}$ has been reported in few others such as Pseudomonas putida IRN22, Acinetobacter pittii IRN19, Micrococcus luteus IRN20 (Montazer et al., 2019) and different species of Pseudomonas, Pseudomonas aeruginosa PAO1, P. aeruginosa ATCC, P. putida, and Pseudomonas syringae (Kyaw et al., 2012), Pseudomonas sp. E4 (Yoon et al., 2012), and bacterial strains from the genera Comamonas, Delftia, and Stenotrophomonas (Peixoto et al., 2017).

\section{Mechanism of PE Degradation}

Aerobic biodegradation of PE by bacteria occurs in four stages: (1) Biodeterioration, which is defined as formation of carbonyl-groups by the action of oxidative enzymes released by microorganisms or induced by exterior agents, like sunlight (ultra-violet) exposure. Subsequent oxidation reduces the number of carbonyl-groups and generates carboxylic acids; (2) Biofragmentation, which involves hydrolysis and/or fragmentation of the polymer carbon chains and the release of intermediate products, mediated by enzymes secreted by microorganisms; (3) Bioassimilation, whereby small hydrocarbon fragments released by biofragmentation are taken-up and metabolized by bacteria or fungi. and (4) Mineralization, transfer the hydrolysis products within the cell wall, intracellular conversion of hydrolysis products to microbial biomass with the associated release of carbon dioxide and water excreted out the cell. Though several groups have reported on biodeterioration and biofragmentation (Albertsson and Karlsson, 1990; Ammala et al., 2011), studies on bioassimilation and complete mineralization of PE are very limited (Yang et al., 2014; Sen and Raut, 2016; Montazer et al., 2019).

Montazer et al. (2019) reported the biofragmentation and bioassimilation of LDPE into biomass by the bacterial species, Pseudomonas putida IRN22, P. putida LS46, Acinetobacter pittii IRN19, and Micrococcus luteus IRN20. These bacterial species were able to utilize the untreated petroleum-derived LDPE as a sole source of carbon and energy for growth 
and generate alkane hydrolysis products as well as accumulate biodegradable polymers in the form of short chain length (scl-) and medium chain length (mcl-) polyhydroxyalkanoates (Montazer et al., 2019). While, A. calcoaceticus and P. aeruginosa were found to metabolize only alkane hydrocarbons and not LPDE (Guzik et al., 2014).

Low-density polyethylene is the most abundant plastic waste discarded in landfills in the form of plastic bags (69.13\%). LDPE is mostly amorphous, with short branches $\left(10-30 \mathrm{CH}_{3}\right.$ per 1,000 carbon atoms) and composed of one or more comonomers, such as 1-butene, 1-hexene, and 1-octene. This branching system makes LDPE chains more accessible and the tertiary carbon atoms at the branch sites more susceptible to attack. The physical arrangement of the polymer chains in LDPE and a lower content of vinylidene defects, which have been shown to be directly correlated with oxidization of the polymer makes it more biodegradable than High-density polyethylene (HDPE). Further, the molar mass of HPDE is much higher, possibly making it more difficult for microorganisms and their oxidizing enzymes to access the polymer chains (Sudhakar et al., 2008; Fontanella et al., 2010). Few comparison studies have been made on the biodegradability of various pre-treated polyethylene materials, including, LDPE, HDPE, and linear low-density polyethylene (LLDPE) films of different thickness by Rhodococcus rhodochrous, which is one of the most efficient bacteria for PE biodegradation (Fontanella et al., 2010). Structural variations in PE polymers formed during polymerization and subsequent processing, such as unsaturated carbon-carbon double bonds, carbonyl groups, and hydroperoxide groups (Ojeda et al., 2011) have been shown to be consumed first by the bacteria resulting in rapid growth.

Many of the species known to degrade PE are capable of hydrolyzing and metabolize linear $n$-alkanes, like paraffin molecules (e.g., $\mathrm{C}_{44} \mathrm{H}_{90}$, Mw 618) (Haines and Alexander, 1974). Alkane hydroxylases (AHs) are the key enzymes involved in aerobic degradation of alkanes by bacteria. The first step involves hydroxylation of C-C bonds to release primary or secondary alcohols, which are oxidized to ketones or aldehydes, and subsequently to hydrophilic carboxylic acids (Alvarez, 2003; Watanabe et al., 2003). Microbial oxidation reduces the number of carbonyl-groups due to the formation of carboxylic acids. Carboxylated $n$-alkanes are analogous to fatty acids, which are catabolized by bacteria via the $\beta$-oxidation system pathway (Restrepo-Flórez et al., 2014). Usha et al. (2011) and Yoon et al. (2012) have demonstrated the microbial oxidation of $n$-alkanes by bacteria via the $\beta$-oxidation pathway and subsequently to the tricarboxylic cycle. The oxidation products released by the action of enzymes in the process may be absorbed by microbial cells where they are catabolized.

Eyheraguibel et al. (2017) revealed that extracellular mechanisms leading to enzymatic oxidation and hydrolysis of chains of PE polymers are also significant They documented oligomers production with maximum 55 carbons (molar mass of $105-850 \mathrm{~g} / \mathrm{mol}$ ) from PE films that adsorbed by bacteria after 240 days of incubation. AHs forms the key enzyme in the alkane hydroxylase system pathway, which are involved in PE degradation in $\beta$-oxidation pathway and known to degrade linear alkanes (Jeon and Kim, 2015). The most important enzymes of interest in the alkane hydroxylase system are the monoxygenases. The number and types of AHs vary greatly in different bacteria, which itself differs in the amount of carbon in the alkane chains (Jeon and Kim, 2016). The Rhodococcus sp. TMP2 genome encodes 5 AHs (alkB1, alkB2, alkB3, alkB4, and alkB5) while the $P$. aeruginosa genome encodes two AHs: alkB1 and alkB2 (Takei et al., 2008). The Alkane hydroxylase system has also been well studied in P. putida GPo1, where the enzyme is involved in the hydroxylation of the terminal carbon-first step of the n-alkane oxidation pathway (Rojo, 2009). Yoon et al. (2012) have shown that AlkB enzyme of $P$. aeruginosa strain E7 played a central role in the mineralization and thus, biodegradation of LMWPE into $\mathrm{CO}_{2}$ (Yoon et al., 2012). The alkB gene was cloned in Pseudomonas sp. E4, and the AlkB enzyme expressed from the recombinant strain participated in the early stage of LMWPE biodegradation, in the absence of the other specific enzymes like rubredoxin and rubredoxin reductase. A class of multicopper enzymes, known as Laccase enzymes (phenol oxidases), expressed by Rodococcus rubber, have also been shown to play an important role in PE biodegradation (Santo et al., 2013).

\section{New Approaches in PE Biodegradation by Invertebrates}

Recent studies have shown that the digestive tracts (gut) of some insects function as bioreactors, with digestive enzymes and gut microbiomes that appear to contribute to and accelerate the biodegradation rate of some recalcitrant plastics. Riudavets et al. (2007) first observed that some insects are able to chew and penetrate PE packaging. Yang et al. (2014) isolated LDPE-degrading bacterial strains, Enterobacter asburiae YT1 and Bacillus sp. YP1 from the gut of Plodia interpunctella (Indianmeal moth). Bacterial isolates identified from the gut of $P$. interpunctella were able to degrade approximately $6-10 \%$ of PE films and released 12 water-soluble products. Yang et al. (2015) reported the complete genome sequence of Bacillus sp. strain YP1, one of isolates from the gut of $P$. interpunctella larvae that was implicated in polyethylene depolymerization and biodegradation. Bombelli et al. (2017) realized the extraordinary ability of larvae of the greater wax moths, Galleria mellonella, to rapidly degrade polyethylene, while producing ethylene glycol (EG). In 2019, Enterobacter sp. D1 was isolated from the gut of G. mellonella by Ren et al. (2019). They confirmed physical changes in the PE-film after treatment, and suggested these physical changes were the result of oxidation reactions caused by the bacteria.

Cassone et al. (2020) assessed the biodegradation of PE by G. mellonella larvae and observed similar results. A diet of LDPE alone enabled subsistence of $G$. mellonella larvae, but was insufficient for growth. These results suppose real biodegradation was not occurred during passing the PE through the gut's larva, but the first biodeterioration or minor oxidation may be taken place resulting in changing in physical properties rather than chemical ones. Billen et al. (2020) showed chewing and ingesting of polyethylene by $T$. molitor larvae created holes and reduced the size of polyethylene films, but digestion was not explicitly confirmed. They suggested that the intestinal microbiomes of these insect larvae play key role in the initial 
short-term biodegradation process, which occurs rapidly within the larval insect gut. However, the insect gut microbiome alone is not sufficient for this initial rapid biodegradation of PE. Rather, both the insect digestive system and the larval gut microbiome are required to achieve accelerated biodegradation of the PE polymers.

In recent years, biodegradation of other petro-plastics, like PE and PS, insect larvae such as Yellow Mealworms (Tenebrio molitor) (Billen et al., 2020), Dark Mealworms (Tenebrio obscurus) (Brandon et al., 2018; Peng et al., 2019), Superworms (Zophobas atratus) (Peng et al., 2020b), Lesser Waxworms (Achroia Grisella) (Kundungal et al., 2019), and by snails (Achatina fulita) (Song et al., 2020), have been reported. Yin et al. (2020) isolated two LDPE-degrading strains from the gut of T. molitor larvae, Acinetobacter sp. strain NyZ450 and Bacillus sp. strain NyZ451. The cells of both strains can depolymerize LDPE but did not grow on it. Their co-culture grew on LDPE and removed LDPE mulching films by $18 \%$ over 30 days. This suggests that biodegradation of LDPE requires multiple microbes. Yang et al. (2021) reported that antibiotic suppression did not stop LDPE depolymerization in the gut of Tenebrio molitor larvae indicating that the digestive enzymes of the larvae are capable of breaking down LDPE.

Despite high rates of biodegradation of PE by live macroorganisms, there are a number of drawbacks that may limit the use of insect larvae as a waste management strategy for petro-plastics like polyethylene. These limitations include: (i) the need to sustain insect cultures to produce the larvae that feed on PE; (ii) the potentially high cost of maintaining these cultures; and (iii) generation of microplastics that may contribute to environmental problems, due to incomplete degradation and lack of mineralization Instead, finding new isolates of bacteria and/or fungi with the ability to degrade PE, and understanding the exact mechanisms of biodegradation pathways, may be more efficient in developing of new methods of PE waste management (Billen et al., 2020; Montazer et al., 2020b).

\section{Polystyrene}

Polystyrene (PS) has been the most abundant plastics produced worldwide and largely manufactured into packaging materials for food and disposable dishware (Plastics Europe, 2017). Like other synthetic plastics, PS is extensively used because of its good mechanical properties and relatively low cost. PS is commonly used in packaging foam, food containers, construction materials (insulation), cassette boxes, compact disks, disposable cups, plates, and cutleries. There are approximately 21 million tons of PS produced worldwide in 2013 (Yang et al., 2015a,b). PS has been grouped into four types of product based on its different applications, General purpose polystyrene (GPPS)/oriented polystyrene (OPS), high impact polystyrene (HIPS), PS foam, and expanded polystyrene (EPS) foam (Ho et al., 2018). PS are extremely stable polymers with high molecular weight and strong hydrophobic character, which makes these polymers highly resistant to biodegradation (Albertsson and Karlsson, 1993; Ho et al., 2018). The carbon-carbon backbone in PS is highly resistant to enzymatic cleavage by oxidation-reduction process (Goldman, 2010).
The biodegradation of PS by several microbes and/or microbial enzymes has been demonstrated (Table 2). PS materials used in these studies range from pure PS or modified PS to PS blended with other polymers. A pure strain of $P$. aeruginosa degraded the modified PS (Shimpi et al., 2012), and Curvularia species was investigated for degradation of atactic PS, without any pretreatment (Motta et al., 2009). The modified PSs used have been PS/PLA composites, and PS/PLA/organically modified montmorillonite (OMMT) blends (soft phyllosilicate minerals that usually form in microscopic crystals, forming clays) (Shimpi et al., 2012). Pure strains of the actinomycete, Rhodococcus ruber have been shown to degrade three forms of PS, pure standard PS flakes, PS powder, and ELISA 96-well microtiter plates produced from pure PS (Mor and Sivan, 2008; Santo et al., 2013). Ward et al. (2006) reported that the P. putida CA-3 (NCIMB 41162) uses styrene oil after pyrolysis as the sole source of carbon and energy to form PHAs. Several investigators have shown that the rate of biodegradation of PS foams and films can be improved by using the polymer-starch blends, which accelerates the structural molecular changes (Jasso et al., 1998; Schlemmer et al., 2009; Pushpadass et al., 2010; Nikolic et al., 2013).

Besides using starch as copolymer, the addition of prooxidants such as metal salt (iron, cobalt, and manganese) to increase biodegradation have also been investigated. It has been found that trace amounts of metals such as $\mathrm{Co}, \mathrm{Mn}, \mathrm{Fe}, \mathrm{Cu}$, and $\mathrm{Ni}$, significantly increase the rate of oxidative degradation (Gorghiu et al., 2004). Prooxidants facilitates cleavage of molecules into smaller fragments containing hydrophilic oxygenated groups that can be easily biodegraded by microorganism in the environment (Shang et al., 2003). Ojeda et al. (2009) reported improved biodegradability of foamed PS when Co and Mn based prooxidant additives were used.

Styrene itself can be used as a carbon source for growth by some microorganisms (Sielicki et al., 1978; Kaplan et al., 1979; Mor and Sivan, 2008; Motta et al., 2009). R. ruber has been reported to form biofilms on PS and partially degrade it (Mor and Sivan, 2008). Styrene catabolism involves the oxidation of styrene to form phenylacetate, which is then converted via the TCA cycle (Di Gennaro et al., 1999). Similar to PE, both laccase and oxidoreductases have been shown to be involved in the biodegradation of PS, such as the AlkB family hydroxylases and hydroquinone peroxidase (Nakamiya et al., 1997; Jeon and Kim, 2015). Xu et al. (2019) has demonstrated the bio-catalytic mechanism of PE and PS degradation by oxidoreductase using quantum mechanism calculations, with the $\mathrm{P} 450$ monooxygenase involved in a typical saturated carbon-carbon bone cleavage reaction (Matthews et al., 2017).

Biodegradation of polystyrene foam (or Styrofoam) has been reported intensively during last 5 years. Yang et al. (2015a,b) found that the larvae of Tenebrio molitor are capable of depolymerizing and mineralizing $\mathrm{PS}$ to $\mathrm{H}_{2} \mathrm{O}$ and $\mathrm{CO}_{2}$ within $24 \mathrm{~h}$, and the degradation is gut microbe dependent based on antibiotic suppression test. The ubiquity of gutmicrobe dependent depolymerization and biodegradation of PS in T. molitor has been confirmed by multiple researchers (Yang et al., 2018a,b, 2021). The same PS biodegradation pattern has also been observed in the members of darkling beetles including 
TABLE 2 | Microorganisms capable of recalcitrant petro-plastics degradation.

\begin{tabular}{|c|c|c|c|c|c|c|}
\hline \multirow{2}{*}{$\begin{array}{l}\text { Examined } \\
\text { polymer } \\
\text { (polymer } \\
\text { under } \\
\text { examination) }\end{array}$} & \multirow[t]{2}{*}{ Species } & \multirow[t]{2}{*}{ Source } & \multirow{2}{*}{$\begin{array}{l}\text { Cultivation } \\
\text { conditions }\end{array}$} & \multicolumn{2}{|c|}{ Polymer degradation } & \multirow[t]{2}{*}{ References } \\
\hline & & & & $\begin{array}{l}\text { In vitro } \\
\text { conditions }\end{array}$ & $\begin{array}{l}\text { Degradation } \\
\text { efficiency }\end{array}$ & \\
\hline \multirow[t]{7}{*}{ Polystyrene } & Pseudomonas sp. & $\begin{array}{l}\text { Soil samples from } \\
\text { plastic dump yard }\end{array}$ & $\begin{array}{l}\text { Mineral medium with } \\
0.85 \% \mathrm{NaCl} \text { and } \mathrm{HIPS} \\
\text { film at } 30^{\circ} \mathrm{C}, 150 \mathrm{rpm}\end{array}$ & $\begin{array}{l}30 \text { days incubation } \\
\text { at } 30^{\circ} \mathrm{C}\end{array}$ & $>10 \%$ weight loss & $\begin{array}{l}\text { Mohan et al., } \\
2016\end{array}$ \\
\hline & Bacillus sp. & & & & $23.7 \%$ weight loss & \\
\hline & $\begin{array}{l}\text { Pseudomonas } \\
\text { aeruginosa }\end{array}$ & $\begin{array}{l}\text { Degraded polymer } \\
\text { nanocomp-osite }\end{array}$ & $\begin{array}{l}\mathrm{NB} \text { medium at } 30^{\circ} \mathrm{C} \text { for } \\
24 \mathrm{~h}\end{array}$ & $\begin{array}{l}28 \text { days incubation } \\
\text { at } 30^{\circ} \mathrm{C} \text { in } \mathrm{MSM}\end{array}$ & $\begin{array}{l}9.9 \% \text { degradation } \\
\text { at } 10 \text { and } 25 \% \text { PS: } \\
\text { PLA composites }\end{array}$ & $\begin{array}{l}\text { Shimpi et al., } \\
2012\end{array}$ \\
\hline & $\begin{array}{l}\text { Pseudomonas } \\
\text { putida CA-3 }\end{array}$ & $\begin{array}{l}\text { Industrial bioreactor } \\
\text { isolate }\end{array}$ & $\begin{array}{l}\text { E2 mineral medium with } \\
67 \mathrm{mg} \text { nitrogen/l and } \\
9.5 \mathrm{mg} / \mathrm{l} \text { styrene oil at } \\
30^{\circ} \mathrm{C}, 200 \mathrm{rpm} \text { for } 24 \mathrm{~h}\end{array}$ & $\begin{array}{l}48 \mathrm{~h} \text { of } \\
\text { fermentation at } \\
30^{\circ} \mathrm{C}, 500 \mathrm{rpm}\end{array}$ & $\begin{array}{l}\text { A single pyrolysis } \\
\text { run and four } \\
\text { fermentation runs } \\
\text { resulted in the } \\
\text { conversion of } 64 \mathrm{~g} \\
\text { of polystyrene to } \\
6.4 \mathrm{~g} \text { of } \mathrm{PHA}\end{array}$ & $\begin{array}{l}\text { Ward et al., } \\
2006\end{array}$ \\
\hline & Curvularia sp. & Soil samples & $\begin{array}{l}\text { Sabouraud's broth at } \\
25^{\circ} \mathrm{C} \text { for } 13 \text { days }\end{array}$ & $\begin{array}{l}9 \text { weeks incubation } \\
\text { at } 25^{\circ} \mathrm{C} \text { in } \\
\text { Sabouraud's agar } \\
\text { embedded with } \\
\text { Ecoflex }\end{array}$ & $\begin{array}{l}\text { Microscopic } \\
\text { examination } \\
\text { showed adherence } \\
\text { and penetrance to } \\
\text { the polymer }\end{array}$ & $\begin{array}{l}\text { Motta et al., } \\
2009\end{array}$ \\
\hline & $\begin{array}{l}\text { Rhodococcus } \\
\text { ruber } \\
\text { Enterobacter sp. } \\
\text { Citrobacter sedlakii } \\
\text { Alcaligenes sp. } \\
\text { Brevundimonas } \\
\text { diminuta }\end{array}$ & Soil samples & $\begin{array}{l}\text { NB medium at } 35^{\circ} \mathrm{C} \text {, } \\
120 \text { rpm for } 10-14 \\
\text { days }\end{array}$ & $\begin{array}{l}8 \text { weeks incubation } \\
\text { at } 35^{\circ} \mathrm{C} \text { in synthetic } \\
\text { medium }\end{array}$ & $0.8 \%$ weight loss & $\begin{array}{l}\text { Mor and Sivan, } \\
2008\end{array}$ \\
\hline & $\begin{array}{l}\text { Exiguobacterium } \\
\text { sp. strain YT2 }\end{array}$ & $\begin{array}{l}\text { Degraded plastic } \\
\text { waste }\end{array}$ & $\begin{array}{l}\text { MSM with e-plastic film } \\
\text { at } 30^{\circ} \mathrm{C}, 150 \text { rpm for } 2 \\
\text { weeks }\end{array}$ & $\begin{array}{l}30 \text { days incubation } \\
\text { at } 30^{\circ} \mathrm{C}, 150 \mathrm{rpm} \text { in } \\
\text { mineral medium }\end{array}$ & $12.4 \%$ weight loss & $\begin{array}{l}\text { Sekhar et al., } \\
2016\end{array}$ \\
\hline \multirow[t]{5}{*}{ Polypropylene } & $\begin{array}{l}\text { Pseudomonas } \\
\text { Vibrio } \\
\text { Aspergillus niger }\end{array}$ & $\begin{array}{l}\text { Plastic dumping } \\
\text { site }\end{array}$ & $\begin{array}{l}\text { Mineral medium (B7) } \\
\text { with } 0.05 \% \text { glucose } \\
\text { and } 0.05 \% \text { sodium } \\
\text { lactate at } 30^{\circ} \mathrm{C}\end{array}$ & $\begin{array}{l}175 \text { days } \\
\text { incubation at } \\
\text { neutral } \mathrm{pH} \text { and } \\
30^{\circ} \mathrm{C}\end{array}$ & $60 \%$ weight loss & $\begin{array}{l}\text { Cacciari et al., } \\
1993\end{array}$ \\
\hline & Bacillus flexus & $\begin{array}{l}\text { Plastic dumping } \\
\text { site }\end{array}$ & $\begin{array}{l}\text { Minimal media with } \\
0.25 \% \text { glucose at } 37^{\circ} \mathrm{C}\end{array}$ & $\begin{array}{l}365 \text { days } \\
\text { incubation at } \\
\text { neutral } \mathrm{pH} \text { and at } \\
35-37^{\circ} \mathrm{C}, 180 \mathrm{rpm}\end{array}$ & $2.5 \%$ weight loss & $\begin{array}{l}\text { Arkatkar et al., } \\
2010\end{array}$ \\
\hline & Bacillus cereus & $\begin{array}{l}\text { Mangrove } \\
\text { sediments }\end{array}$ & $\begin{array}{l}\text { Mineral salt medium at } \\
29^{\circ} \mathrm{C}\end{array}$ & $\begin{array}{l}40 \text { days incubation } \\
\text { at } 33^{\circ} \mathrm{C}, 150 \mathrm{rpm}\end{array}$ & $12 \%$ weight loss & $\begin{array}{l}\text { Auta et al., } \\
2017\end{array}$ \\
\hline & $\begin{array}{l}\text { Sporosarcina } \\
\text { globispora }\end{array}$ & & & & $11 \%$ weight loss & \\
\hline & Bacillus sp. & $\begin{array}{l}\text { Municipal compost } \\
\text { waste }\end{array}$ & Minimal media at $37^{\circ} \mathrm{C}$ & $\begin{array}{l}15 \text { days incubation } \\
\text { at } 37^{\circ} \mathrm{C}, 120 \mathrm{rpm}\end{array}$ & $\begin{array}{l}10-12 \% \text { weight } \\
\text { loss }\end{array}$ & $\begin{array}{l}\text { Jain et al. } \\
(2018)\end{array}$ \\
\hline \multirow[t]{2}{*}{$\begin{array}{l}\text { Polyvinyl } \\
\text { chloride }\end{array}$} & White rot fungi & $\begin{array}{l}\text { Department of } \\
\text { Agriculture Forest } \\
\text { Products Lab. } \\
\text { Madison, } \\
\text { Wisconsin, } \\
\text { United States }\end{array}$ & $\begin{array}{l}\text { Liquid medium at } 30^{\circ} \mathrm{C} \text {, } \\
\mathrm{pH} 4.5 \text { and } 150 \text { rpm for } \\
7 \text { days }\end{array}$ & $\begin{array}{l}30 \text { days incubation } \\
\text { at } 30^{\circ} \mathrm{C}\end{array}$ & $\begin{array}{l}\text { Not specified } \\
\text { (intrinsic viscosity of } \\
\text { the degraded } \\
\text { polymer films } \\
\text { decreased) }\end{array}$ & $\begin{array}{l}\text { Klrbas et al., } \\
1999\end{array}$ \\
\hline & $\begin{array}{l}\text { Trichocladium sp. } \\
\text { Chaetomium sp. }\end{array}$ & Soil samples & $\begin{array}{l}\text { ISP2 (Difco) medium at } \\
25^{\circ} \mathrm{C} \text { for } 7 \text { days }\end{array}$ & $\begin{array}{l}90 \text { and } 180 \text { days } \\
\text { incubation at } 30^{\circ} \mathrm{C}\end{array}$ & $\begin{array}{l}1 \text { or } 0.4 \% \text { after } 3 \text { or } \\
6 \text { months, } \\
\text { respectively }\end{array}$ & $\begin{array}{l}\text { Kaczmarek and } \\
\text { Bajer, } 2007\end{array}$ \\
\hline
\end{tabular}


TABLE 2 | Continued

\begin{tabular}{|c|c|c|c|c|c|c|}
\hline \multirow{2}{*}{$\begin{array}{l}\text { Examined } \\
\text { polymer } \\
\text { (polymer } \\
\text { under } \\
\text { examination) }\end{array}$} & \multirow[t]{2}{*}{ Species } & \multirow[t]{2}{*}{ Source } & \multirow[t]{2}{*}{$\begin{array}{l}\text { Cultivation } \\
\text { conditions }\end{array}$} & \multicolumn{2}{|c|}{ Polymer degradation } & \multirow[t]{2}{*}{ References } \\
\hline & & & & In vitro conditions & $\begin{array}{l}\text { Degradation } \\
\text { efficiency }\end{array}$ & \\
\hline & $\begin{array}{l}\text { Pseudomonas } \\
\text { citronellolis } \\
\text { Bacillus flexus }\end{array}$ & $\begin{array}{l}\text { Leibniz Institute } \\
\text { DSMZ-German } \\
\text { Collection of } \\
\text { Microorganisms } \\
\text { and Cell Cultures } \\
\text { (Germany) }\end{array}$ & $\begin{array}{l}\text { MSM with } 20 \mathrm{~g} / \mathrm{l} \\
\text { glucose at } 30^{\circ} \mathrm{C} \text {, } \\
150 \mathrm{rpm}\end{array}$ & $\begin{array}{l}90 \text { days incubation at } \\
30 \text { and } 37^{\circ} \mathrm{C}, 150 \mathrm{rpm}\end{array}$ & $\begin{array}{l}19 \% \text { after } 30 \text { days } \\
\text { incubation }\end{array}$ & $\begin{array}{l}\text { Giacomucci } \\
\text { et al., } 2019\end{array}$ \\
\hline & $\begin{array}{l}\text { Pseudomonas } \\
\text { aeruginosa }\end{array}$ & $\begin{array}{l}\text { Activated sludge } \\
\text { (waste water } \\
\text { treatment plant) }\end{array}$ & $\begin{array}{l}\mathrm{MSM} \text { with }\left(\mathrm{NH}_{4}\right)_{2} \mathrm{SO}_{4} \\
0.67 \mathrm{~g} / \text { liter at } 23^{\circ} \mathrm{C}, \\
150 \mathrm{rpm}\end{array}$ & $\begin{array}{l}24 \text { days incubation } \\
23^{\circ} \mathrm{C}\end{array}$ & $\begin{array}{l}\text { VC concentrations as } \\
\text { high as } 7.3 \mathrm{mM} \text { were } \\
\text { biodegraded }\end{array}$ & $\begin{array}{l}\text { Verce et al., } \\
2000\end{array}$ \\
\hline & $\begin{array}{l}\text { Pseudomonas } \\
\text { otitidis } \\
\text { Bacillus cereus } \\
\text { Acanthopleuro- } \\
\text { bacter pedis } \\
\text { Aspergillus } \\
\text { fumigatus } \\
\text { Aspergillus niger } \\
\text { Aspergillus sydowii } \\
\text { Phanerochaete } \\
\text { chrysosporium } \\
\text { Lentinus tigrinus }\end{array}$ & Soil samples & $\mathrm{MSM}$ at $30^{\circ} \mathrm{C}, 150 \mathrm{rpm}$ & $\begin{array}{l}90 \text { days incubation at } \\
30^{\circ} \mathrm{C}\end{array}$ & $\begin{array}{l}\text { Clear surface } \\
\text { aberrations and } \\
\text { disintegration in PVC } \\
\text { films. Significant } \\
\text { decrease in the } \\
\text { molecular weight of film } \\
\text { from } 80,275 \text { to } 78,866 \\
\mathrm{Da}\end{array}$ & \\
\hline \multirow[t]{8}{*}{$\begin{array}{l}\text { Polyethylene } \\
\text { terephthalate }\end{array}$} & Ideonella sakaiensis & $\begin{array}{l}\text { PET recycling } \\
\text { factory }\end{array}$ & $\begin{array}{l}\text { NB medium with PET } \\
\text { at } 30^{\circ} \mathrm{C}\end{array}$ & $\begin{array}{l}42 \text { days incubation at } \\
30^{\circ} \mathrm{C}\end{array}$ & $\begin{array}{l}\text { Almost complete } \\
\text { degradation achieved }\end{array}$ & $\begin{array}{l}\text { Yoshida et al., } \\
2016\end{array}$ \\
\hline & Bacillus subtilis & $\begin{array}{l}\text { TU-Graz culture } \\
\text { collection }\end{array}$ & $\begin{array}{l}\text { NB medium at } 30^{\circ} \mathrm{C} \text {, } \\
125 \mathrm{rpm}\end{array}$ & $\begin{array}{l}24 \mathrm{~h} \text { incubation at } 30^{\circ} \mathrm{C} \\
\text { and } \mathrm{pH} 7.0\end{array}$ & $\begin{array}{l}\text { Not specified } \\
\text { (degradation products } \\
\text { determined by } \\
\text { LC-MS/MS analysis) }\end{array}$ & $\begin{array}{l}\text { Ribitsch et al., } \\
2011\end{array}$ \\
\hline & $\begin{array}{l}\text { Pseudomonas } \\
\text { putida G016 }\end{array}$ & $\begin{array}{l}\text { National Collection } \\
\text { of Industrial, Food } \\
\text { and Marine } \\
\text { Bacteria (NCIMB) }\end{array}$ & $\begin{array}{l}\text { MSM medium with } \\
\text { sodium terephthalate } \\
\text { produced from a PET } \\
\text { pyrolysis product and } \\
\text { waste glycerol from } \\
\text { biodiesel at } 30^{\circ} \mathrm{C} \text {, } \\
200 \text { rpm for } 24 \mathrm{~h}\end{array}$ & $48 \mathrm{~h}$ incubation at $30^{\circ} \mathrm{C}$ & $\begin{array}{l}\text { PHA productivity }(\mathrm{g} / \mathrm{l} / \mathrm{h}) \\
1.8 \text { - to } 2.2 \text {-fold }\end{array}$ & $\begin{array}{l}\text { Kenny et al., } \\
2012\end{array}$ \\
\hline & $\begin{array}{l}\text { Thermomonospora } \\
\text { fusca }\end{array}$ & $\begin{array}{l}\text { 6-month-old } \\
\text { mature compost } \\
\text { from green waste } \\
\text { (compost plant) }\end{array}$ & $\begin{array}{l}\text { MSM medium at } 55^{\circ} \mathrm{C} \\
\text { for } 14 \text { days }\end{array}$ & $\begin{array}{l}14 \text { days incubation at } \\
55^{\circ} \mathrm{C} \text { on MSV agar }\end{array}$ & $\begin{array}{l}\text { Degradation rates of } \\
20 \mathrm{mg} / \text { week } \mathrm{cm}^{-2}\end{array}$ & $\begin{array}{l}\text { Kleeberg et al., } \\
1998\end{array}$ \\
\hline & $\begin{array}{l}\text { Thermobifida alba } \\
\text { AHK119 }\end{array}$ & $\begin{array}{l}\text { Composted } \\
\text { polyester films. } \\
\text { IPOD of AIST, } \\
\text { Tsukuba, Japan) }\end{array}$ & $\begin{array}{l}\text { LB medium containing } \\
\text { the hydrolyzed polymer } \\
\text { suspension at } 50^{\circ} \mathrm{C} \text { for } \\
3 \text { days }\end{array}$ & $\begin{array}{l}14 \text { days incubation at } \\
50^{\circ} \mathrm{C}\end{array}$ & $\begin{array}{l}\text { Zone of clearance was } \\
\text { observed }\end{array}$ & Hu et al., 2010 \\
\hline & $\begin{array}{l}\text { Thermomonospora } \\
\text { curvata }\end{array}$ & $\begin{array}{l}\text { Composts } \\
\text { containing plant } \\
\text { materials }\end{array}$ & $\begin{array}{l}\text { MSM medium at } 50^{\circ} \mathrm{C} \\
\text { at a wide range of } \mathrm{pH} \\
\text { from } 7.5 \text { to } 11 \text { for } 3 \\
\text { days }\end{array}$ & $\begin{array}{l}7 \text { days incubation at } \mathrm{pH} \\
8.5 \text { and } 50^{\circ} \mathrm{C}, 55 \text { and } \\
60^{\circ} \mathrm{C}\end{array}$ & $\begin{array}{l}\text { At } 50^{\circ} \mathrm{C} \text {, hydrolysis rate } \\
3.3 \times 10^{-3} \mathrm{~min}^{-1}\end{array}$ & $\begin{array}{l}\text { Wei et al., } \\
2014 b\end{array}$ \\
\hline & $\begin{array}{l}\text { Thermobifida } \\
\text { halotolerans }\end{array}$ & $\begin{array}{l}\text { German Resource } \\
\text { Centre for } \\
\text { Biological Material } \\
\text { (DSMZ, Germany) }\end{array}$ & $\begin{array}{l}\text { LB medium at } 37^{\circ} \mathrm{C} \text {, } \\
160 \text { rpm for } 24 \mathrm{~h}\end{array}$ & $\begin{array}{l}2 \mathrm{~h} \text { incubation at } 50^{\circ} \mathrm{C} \text {, } \\
350 \mathrm{rpm} \text { and } \mathrm{pH} 7.0\end{array}$ & $\begin{array}{l}\text { The amount of MHET } \\
\text { and TA released were } \\
\text { around } 19.8 \text { and } 21.5 \\
\text { mmol/mol of enzyme, } \\
\text { respectively }\end{array}$ & $\begin{array}{l}\text { Ribitsch et al., } \\
2012 a\end{array}$ \\
\hline & $\begin{array}{l}\text { Saccharomonospora } \\
\text { viridis }\end{array}$ & $\begin{array}{l}\text { Compost } \\
\text { (Okayama, Japan) } \\
\text { IPOD of the NITE }\end{array}$ & $\begin{array}{l}\text { LB medium at } 50^{\circ} \mathrm{C} \\
\text { under shaking for } \\
12-16 \mathrm{~h}\end{array}$ & $\begin{array}{l}3 \text { days incubation at } \\
63^{\circ} \mathrm{C} \text { under shaking }\end{array}$ & $\begin{array}{l}\text { 13.5\% weight loss for } \\
\text { PET-GF and } 27.0 \% \text { for } \\
\text { PET-S }\end{array}$ & $\begin{array}{l}\text { Kawai et al., } \\
2014\end{array}$ \\
\hline
\end{tabular}


TABLE 2 | Continued

\begin{tabular}{|c|c|c|c|c|c|c|}
\hline \multirow{2}{*}{$\begin{array}{l}\text { Examined } \\
\text { polymer } \\
\text { (polymer } \\
\text { under } \\
\text { examination) }\end{array}$} & \multirow[t]{2}{*}{ Species } & \multirow[t]{2}{*}{ Source } & \multirow{2}{*}{$\begin{array}{l}\text { Cultivation } \\
\text { conditions }\end{array}$} & \multicolumn{2}{|c|}{ Polymer degradation } & \multirow[t]{2}{*}{ References } \\
\hline & & & & In vitro conditions & $\begin{array}{l}\text { Degradation } \\
\text { efficiency }\end{array}$ & \\
\hline \multirow[t]{8}{*}{ Polyurethane } & $\begin{array}{l}\text { Pseudomonas } \\
\text { fluorescens }\end{array}$ & $\begin{array}{l}\text { National Research } \\
\text { Laboratory, } \\
\text { Washington D.C. }\end{array}$ & $\begin{array}{l}\text { YES medium } \\
\text { containing Impranil at } \\
30^{\circ} \mathrm{C} \text { for } 5 \text { days }\end{array}$ & $\begin{array}{l}24 \mathrm{~h} \text { incubation at } 30^{\circ} \mathrm{C} \\
\text { using polyurethane } \\
\text { plates }\end{array}$ & $\begin{array}{l}\text { Zone of clearance was } \\
\text { observed }\end{array}$ & $\begin{array}{l}\text { Howard and } \\
\text { Blake, } 1998\end{array}$ \\
\hline & $\begin{array}{l}\text { Pseudomonas } \\
\text { chlororaphis }\end{array}$ & $\begin{array}{l}\text { Microbial } \\
\text { consortium from } \\
\text { the Naval Research } \\
\text { Laboratory, } \\
\text { Washington, DC, } \\
\text { United States }\end{array}$ & $\begin{array}{l}\text { LB medium at } 30^{\circ} \mathrm{C} \text {, } \\
180 \mathrm{rpm}\end{array}$ & $\begin{array}{l}6 \mathrm{~h} \text { incubation at } 23^{\circ} \mathrm{C} \\
\text { on plates with Impranil }\end{array}$ & $\begin{array}{l}\text { Zone of clearance was } \\
\text { observed }\end{array}$ & $\begin{array}{l}\text { Stern and } \\
\text { Howard, } 2000\end{array}$ \\
\hline & $\begin{array}{l}\text { Exophiala } \\
\text { jeanselmei }\end{array}$ & $\begin{array}{l}\text { Soil samples from a } \\
\text { polyurethane } \\
\text { factory in Japan }\end{array}$ & $\begin{array}{l}\text { Difco Sabouraud liquid } \\
\text { medium at } 25^{\circ} \mathrm{C} \text { for } \\
6-8 \text { days }\end{array}$ & $\begin{array}{l}7 \text { days incubation at } \\
25^{\circ} \mathrm{C} \text { with } \\
\text { tolylcarbamate } \\
\text { compounds that } \\
\text { resemble the urethane } \\
\text { segments in TDI-based } \\
\text { polyurethanes }\end{array}$ & $\begin{array}{l}\text { Able to hydrolyze the } \\
\text { urethane in } \\
\text { tolylcarbamate } \\
\text { compounds }\end{array}$ & $\begin{array}{l}\text { Owen et al., } \\
1996\end{array}$ \\
\hline & $\begin{array}{l}\text { Aureobasidium } \\
\text { pullulans } \\
\text { Cladosporium sp. } \\
\text { Curvularia } \\
\text { senegalensis } \\
\text { Fusarium solani }\end{array}$ & $\begin{array}{l}\text { Garden soil near } \\
\text { Washington, D.C. }\end{array}$ & $\begin{array}{l}\text { YES medium } \\
\text { containing Impranil at } \\
25^{\circ} \mathrm{C} \text { for } 6 \text { days }\end{array}$ & $\begin{array}{l}\text { Several days to weeks } \\
\text { incubation at } 25^{\circ} \mathrm{C} \text { in } \\
\text { YES-PG agar plates }\end{array}$ & $\begin{array}{l}\text { Zone of clearance was } \\
\text { observed }\end{array}$ & $\begin{array}{l}\text { Crabbe et al., } \\
1994\end{array}$ \\
\hline & $\begin{array}{l}\text { Pseudomonas } \\
\text { aeruginosa } \\
\text { Corynebacterium } \\
\text { sp. }\end{array}$ & $\begin{array}{l}\text { River mud enriched } \\
\text { with various } \\
\text { platicisers }\end{array}$ & $\begin{array}{l}\text { MSM with } 1 \% \text { yeast } \\
\text { extract }\end{array}$ & $\begin{array}{l}12 \text { weeks incubation at } \\
25^{\circ} \mathrm{C} \text { in MSM medium } \\
\text { with yeast extract }\end{array}$ & $\begin{array}{l}9.30+1.32 \% \text { weight } \\
\text { loss } 15.77 \pm 3.5 \\
\% \text { weight loss }\end{array}$ & Kay et al., 1991 \\
\hline & $\begin{array}{l}\text { Comamonas } \\
\text { acidovorans }\end{array}$ & $\begin{array}{l}\text { Soil samples of } \\
\text { Tsukuba City in } \\
\text { Japan }\end{array}$ & $\begin{array}{l}\text { Basal mineral medium } \\
\text { with polyurethane at } \\
30^{\circ} \mathrm{C}, 120 \mathrm{rpm} \text { for } 14 \\
\text { days }\end{array}$ & $\begin{array}{l}7 \text { days incubation with } \\
\text { PUR films at } 30^{\circ} \mathrm{C}\end{array}$ & $\begin{array}{l}\text { About } 48 \% \text { of the } \\
\text { added PUR was } \\
\text { degraded }\end{array}$ & $\begin{array}{l}\text { Nakajima- } \\
\text { Kambe et al., } \\
1995\end{array}$ \\
\hline & $\begin{array}{l}\text { Acinetobacter } \\
\text { calcoaceticus } \\
\text { Arthrobacter } \\
\text { globiformis }\end{array}$ & $\begin{array}{l}\text { Oil-contaminated } \\
\text { Connecticut soil }\end{array}$ & $\begin{array}{l}\text { Tripticase soy broth at } \\
30^{\circ} \mathrm{C}, 150 \mathrm{rpm} \text { for } 24 \mathrm{~h}\end{array}$ & $\begin{array}{l}10 \text { days incubation at } \\
30^{\circ} \mathrm{C} \text { in MSM with } \\
\text { polyurethane painted } \\
\text { aluminum coupons }\end{array}$ & $\begin{array}{l}\text { Release of pigment } \\
\text { from the polyurethane } \\
\text { coatings into the broth } \\
\text { was observed in } 192 \mathrm{~h}\end{array}$ & $\begin{array}{l}\text { El-Sayed et al., } \\
1996\end{array}$ \\
\hline & Bacillus subtilis & $\begin{array}{l}\text { Soil sample from a } \\
\text { mecocosm study }\end{array}$ & $\begin{array}{l}\text { YES medium at at } \\
30^{\circ} \mathrm{C}, 150 \mathrm{rpm} \text { for } 12 \mathrm{~h}\end{array}$ & $\begin{array}{l}24 \mathrm{~h} \text { incubation at } 30^{\circ} \mathrm{C} \\
\text { on a LB agar plate with } \\
\text { Impranil }\end{array}$ & $\begin{array}{l}\text { Zone of clearance was } \\
\text { observed }\end{array}$ & $\begin{array}{l}\text { Rowe and } \\
\text { Howard, } 2002\end{array}$ \\
\hline
\end{tabular}

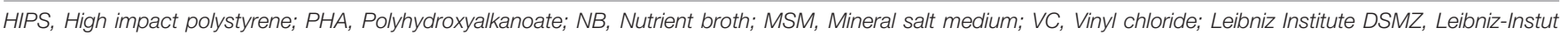

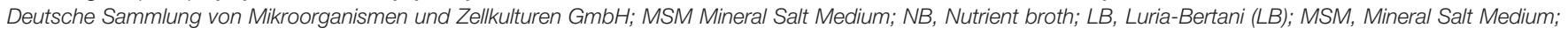

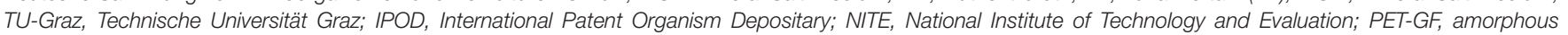

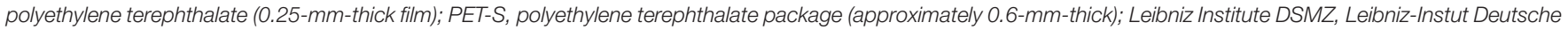

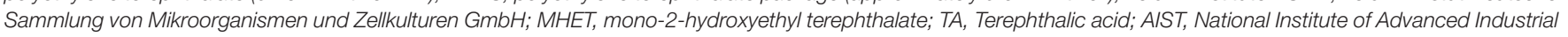
Science and Technology; YES, Yeast-extract salts medium; YES-PG, Yeast-extract salts medium with $3 \mathrm{mg} / \mathrm{ml} \mathrm{of} \mathrm{Impranil}$ and $4 \mathrm{mg} / \mathrm{ml}$ of gelatin.

Tenebrio obscurus (Peng et al., 2019) and Zophobas atratus (Peng et al., 2020b). PS-degrading bacterial cultures of Exiguobacterium sp. strain TY2 was isolated from the gut of $T$. molitor larvae (Yang et al., 2015b) while Pseudomonas aeruginosa strain DSM 50071 (Kim et al., 2020) was isolated from Z. atratus. The mass reduction rates by the bacterial cultures were much slower than that occurred in the gut of the larvae, suggesting that the synergetic biodegradation occurs in the digestive systems. In addition, PS depolymerization with limited extent pattern was also observed in the digestive intestines of land snails, Achatina fulica (Song et al., 2020) and larvae of Galleria mellonella (Lou et al., 2020). Antibiotic suppression with oxytetracycline did not stop PS depolymerization in the snails, suggesting the presence of PS-degrading enzyme(s).

\section{Polypropylene}

Polypropylene (PP), expressed as $\mathrm{C}_{n} \mathrm{H}_{2 n}$, is also the most widely used linear hydrocarbon polymers among the synthetic polymers. PP has a methyl-group in place of one of the hydrogens present in $\mathrm{PE}$ on every other carbon, which gives rise to the presence 
of three stereoisomeric forms namely, atactic, isotactic, and syndiotactic (Baker and Mead, 2002). This polymer was first synthesized by J. Paul Hogan and Robert L. Banks in 1951 with propylene as the monomer (Stinson, 1987). Metallocene catalysts have also been used for its synthesis. Its properties are similar to polyethylene, but it is slightly harder and more heat resistant and has a high chemical resistance Polypropylene is the secondmost widely produced commodity plastics (after polyethylene) and it is often used in packaging and labeling. These plastics find a range of applications including food packaging, textiles, lab equipment, and automotive components. PP are grouped as polyolefins together with PE, defined as inert materials not prone to microbial attack because of the hydrophobic backbones composed of long carbon chains, high molecular weight (from 10,000 to $40,000 \mathrm{~g} / \mathrm{mol}$ ) and the added antioxidants and stabilizers during their manufacture which prevents polyolefins from atmospheric oxidation (Zheng and Yanful, 2005). However, substitution of methyl in place of hydrogen in the $\beta$-position makes it more resistant to microbial attack (Zuchowska et al., 1998, 1999).

Very few studies of PP biodegradation have been reported (Table 2). Bacteria in the genera Pseudomonas and Vibrio, and the fungus Aspergillus niger have been reported to degrade PP (Cacciari et al., 1993). Most of the studies have been carried out using pretreated PP. The pretreatment techniques involved $\gamma$ irradiation (Iwamoto and Tokiwa, 1994), UV-irradiation (Huang et al., 2005; Kaczmarek et al., 2005; Sameh et al., 2006), or thermal treatment (Ramis et al., 2004) and have been shown to reduce the hydrophobicity of the polymer or introduces groups such as $\mathrm{C}=\mathrm{O}$ or $-\mathrm{OH}$, which are more susceptible to degradation. Formation of new groups (carbonyl and hydroxyl) and a decrease in viscosity have been observed during the degradation process (Iwamoto and Tokiwa, 1994; Sameh et al., 2006). Bacillus flexus has been shown to biodegrade UV-pretreated PP (Arkatkar et al., 2010). Biodegradation of polypropylene have been improved by using polymer blends with carbohydrates, starch or cellulose blends like that reported for polyethylene and polystyrene. The use of the blends facilitates adhesion of the microorganisms to the surface of the polymer and acts as a co-metabolite (Cacciari et al., 1993; Zuchowska et al., 1998; Ramis et al., 2004; Kaczmarek et al., 2005; Morancho et al., 2006). Biodegradation of Polycaprolactone (PCL) blended PP has also been demonstrated using lipase since lipase is well known to degrade the ester linkages of PCL (Weiland et al., 1995).

\section{Polyvyinylchloride}

Poly(vinyl chloride) (PVC) is a synthetic polymer well known for many years and are widely used in its rigid or plasticized form (Braun, 2004). However, the pollution caused by waste PVCbased plastics is a serious problem. Many studies have reported on the thermal and photo degradation of PVC (Braun, 1975; Owen, 1976, 2012; Decker, 1984; Seppala et al., 1991). Torikai and Hasegawa (1999) reported accelerated photodegradation of PVC when exposed to short-wavelength radiation. However, biodegradation of PVC has been attempted in very few studies (Klrbas et al., 1999; Kaczmarek and Bajer, 2007; Ali et al., 2014; Giacomucci et al., 2019) and shown by very few microorganisms
(Table 2). White rot fungi in the Basidiomycotina were reported to biodegrade low molecular weight PVC when subjected to nutrient (nitrogen, carbon or sulfur) limiting conditions (Klrbas et al., 1999).

The ability of the white rot fungi to degrade the organopollutants to carbon dioxide, has been shown to be dependent on a nonspecific, non-stereo-selective lignin degrading system. Lignin is a complex heteropolymer and is probably the most-difficult-to degrade naturally occurring organic compound. The lignin degrading system includes a group of peroxidases known as Ligninases, which catalyze the initial oxidative depolymerization of lignin polymers (Bumpus and Aust, 1987; Bumpus et al., 1988; Shah et al., 1992). The growth of Trichocladium sp. and Chaetomium sp. was accelerated in the presence of cellulose with PVC as a carbon source, suggesting co-meatbolims of PVC with the cellulose (Kaczmarek and Bajer, 2007). Also, additives introduced into plastics such as plasticizers, contribute to fungal nutrients for growth.

Pseudomonas citronellolis and Bacillus flexus have also been found to biodegrade PVC film, with high depolymerizing activity toward PVC additives relative to the PVC polymer chains (Giacomucci et al., 2019). These strains have been shown to form dense biofilm on the plastic film surface and cause a decrease in the mean molecular weight of the PVC film. A dense biofilm and a decrease in mean molecular weight (Mn) has been reported as proof of polymer chain biodegradation (Das et al., 2012; Wilkes and Aristilde, 2017; Syranidou et al., 2017; Ahmed et al., 2018). Decreases in molecular weight can be attributed to the action of exocellular enzymes released into the culture medium, which causes the hydrolysis of polymers at the ends of backbone chains as well as within the chains (Wilkes and Aristilde, 2017). Pseudomonas putida strain AJ was reported to use vinyl chloride monomers as carbon source for growth (Verce et al., 2000). The composting and biodegradation processes of PVC are still controversial because of the possible formation of degradation products containing chlorine, which are not neutral for the environment.

Peng et al. (2020a) found that T. molitor larvae are capable of depolymerizing and biodegrading PVC materials rapidly to organic chlorinated intermediates but mineralization extent was very limited with only $2.9 \%$ of PVC conversion to chloride. The depolymerization stopped when gut microbes were suppressed with antibiotic gentamicin, indicating gut-microbial dependence, similar to observations recorded for PS degradation by T. molitor larvae.

\section{PETRO-PLASTICS WITH ESTER-BOND BACK-BONES AND SIDE-CHAINS}

Polyethylene terephthalate (PET) and polyurethane (PU) plastics have heteroatoms in the main chain. Plastics composed of polymers with carbon and hetero atoms in the main chain have improved thermal stability relative to polymers with only carbon backbone (Venkatachalam et al., 2012). These polymers are susceptible to hydrolytic attack of e.g., ester or amide bonds (Muller et al., 2001). Plastics with heteroatoms in the 


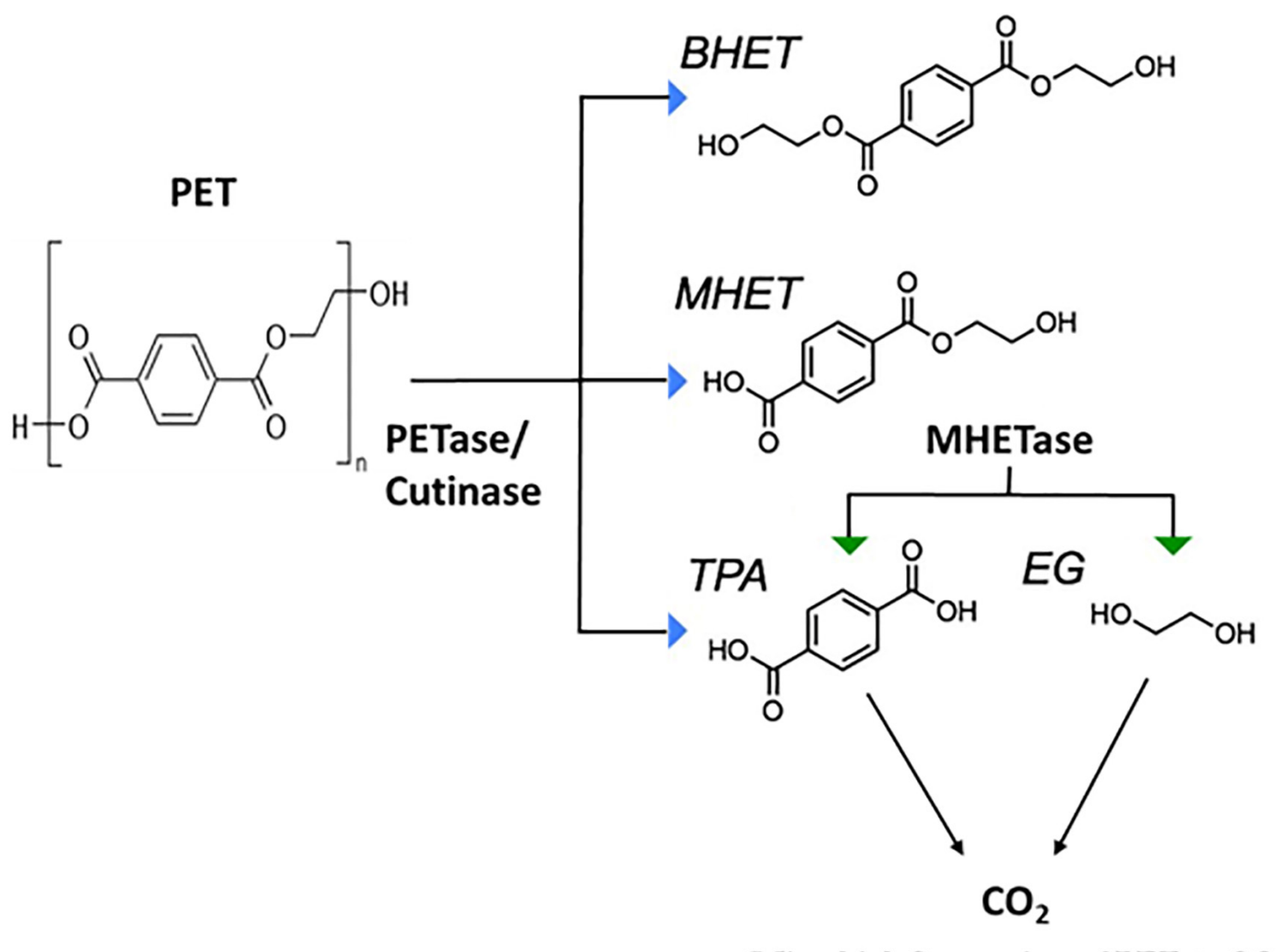

(Microbial Conversion of PET to $\mathrm{CO}_{2}$ )

FIGURE 4 | Microbial degradation of Polyethylene Terephthalate (PET) (adapted from Austin et al., 2018). PETase, polyethylene terephthalate (PET) hydrolase or PET-digesting enzyme; BHET, bis(2-hydroxyethyl) terephthalic acid; MHET, mono(2-hydroxyethyl) terephthalic acid; TPA, terephthalic acid; EG, Ethylene glycol.

main chain can be degraded by photo-oxidation, hydrolysis and biodegradation (Muller et al., 2001). This results in the formation of smaller fragments and carboxylic end groups.

\section{Polyethylene Terephthalate PET Degrading Microorganisms}

Polyethylene terephthalate is one of the major synthetic petroplastics (Figure 1) that is produced in very large amounts globally. Its worldwide production accounted to 56 million tons in 2013 (Neufeld et al., 2016). PET consists of aromatic polyesters with high glass transition temperatures ( $\mathrm{Tg}$ ) of approximately $75-80^{\circ} \mathrm{C}$ in air. However, $\mathrm{Tg}$ decreases to $60-65^{\circ} \mathrm{C}$ in aqueous solution (Kawai et al., 2014). At temperatures above the Tg, the amorphous regions of PET become flexible and more accessible to microbial degradation and/or enzymatic attack. With polymer degradation, a decrease in $\mathrm{Tg}$ was observed as a result of reduction in the average chain length, due to the higher motility of shorter chains (Odusanya et al., 2013).

PET is used in a wide-variety of applications, such as in manufacturing bottles, containers, textile fibers, and films. PET polymers differ in crystallinities based on its usage. While, most PET used for manufacturing textiles and bottles has high crystallinity (30-40\%), PET used for packaging has less crystallinity (approximately 8\%) (Kawai et al., 2014). Commercially available low-crystalline PET (PET-GF) has approximately 6-7\% crystallinity (Ronqvist et al., 2009; Kawai et al., 2014).

Although most biodegradable plastics are polyesters [e.g., polyhydroxyalkanoate, PCL, polybutylene succinate, polybutylene succinate-co-adipate, and poly(butylene adipateco-terephthalate) (PBAT)], PET, which is also a polyester, but is considered to be recalcitrant to biodegradation (MarquesCalvo et al., 2006). However, several microorganisms capable of metabolizing these polymers have been identified in recent years (Table 2). These include the bacterium Ideonella sakaiensis 201-F6, is able to depolymerize PET polymers and utilize the terephthalate subunits as a carbon and energy source for metabolism and growth (Yoshida et al., 2016). Biotechnological conversion of PET through pyrolysis and conversion to polyhydroxyalkanoate has been demonstrated using different Pseudomonads (Ward et al., 2006; Kenny et al., 2008; Guzik et al., 2014). Pyrolysis of PET resulted in terephthalate, which was used as feedstock for P. putida GO16 (Kenny et al., 2012). The degradation rate of PET films depends on the crystallinity, purity of films and orientation of the polymer chains. The degradation of the commercially available standard PET film 
(pure and amorphous PET) at $50^{\circ} \mathrm{C}$ was found significantly lower (approximately 5\%), however, the degradation increased with high temperatures $\left(55,60\right.$, and $65^{\circ} \mathrm{C}$ ) to more than $30 \%$ (Oda et al., 2018).

\section{Mechanism of PET Biodegradation}

Yoshida et al. (2016) demonstrated that PET-digesting enzyme labeled as PETase, converts PET to mono(2-hydroxyethyl) terephthalic acid (MHET), with minimal amounts of terephthalic acid (TPA) and bis(2-hydroxyethyl)-TPA as secondary products (Figure 4). Another enzyme, MHETase (MHET-digesting enzyme), further hydrolyzes MHET into the two monomers, TPA and EG (Figure 4). Li et al. (2019) has reported the metabolism of EG, the second component of PET besides terephthalate, in P. putida KT2440. The metabolism of EG and its derivatives has resulted in different oxidation products such as glycolaldehyde, glyoxal, glycolate, and glyoxylate (Figure 5), which have a variety of value-added applications. These find use as reactive building blocks in the production of agro-, aroma-, and polymer chemicals, or pharmaceuticals (Sajtos, 1991; Mattioda and Christidis, 2000; Yue et al., 2012). Several microorganisms have been reported to utilize EG such as those from Acetobacter and Gluconobacter (DeLey and Kersters, 1964), Acinetobacter and halophilic bacterium, T-52 (ATCC 27042) (Gonzalez et al., 1972; Caskey and Taber, 1981), Flavobacterium species (Child and Willetts, 1978), Hansenula (Harada and Hirabayashi, 1968), Candida, Pichia naganishii AKU4267, and Rhodotorula sp. 3Pr-126 (Kataoka et al., 2001).

The genetic mechanism for the pathways enabling EG metabolism has been well demonstrated in P. putida. P. putida strain JM37 was able to utilize EG as a sole source of carbon and energy. However, while P. putida KT2440 was able to use EG a carbon source, it did not grow well. Both strains were able to metabolize EG and produced glycolic acid and glyoxylic acid (Figure 5). Initially, the diol is oxidized into glyoxylate in a series of reactions catalyzed by a set of dehydrogenases genes that encode the PP_0545, PedI, PedE, and PedH enzymes (Muckschel et al., 2012; Wehrmann et al., 2017). The conversion of EG to glyoxylate yields three reducing equivalents, either in the form of $\mathrm{PQQH}_{2}, \mathrm{NADH}$, or in a direct coupling to the electron transport chain. Glyoxylate can be further metabolized by the Glyoxylate carboligase B (GclB) enzyme or AceA enzyme involved in the glyoxylate shunt (Blank et al., 2008) yielding two molecules of $\mathrm{CO}_{2}$ and two reducing equivalents.

In $P$. putida strain JM37, the activity of two additional pathways, namely, Gcl and GlcB, leads to rapid metabolism of EG without accumulation of the intermediates and/or oxalic acid (Muckschel et al., 2012). In case of $P$. putida U and $P$. aeruginosa, the Pyrroloquinoline quinone (PQQ)-dependent alcohol dehydrogenases involved are the PedE and PedH, and the ExaA (formerly QedH) enzymes (Arias et al., 2008). These periplasmic enzymes have been found essential for growth utilizing ethanol as a carbon source, since it catalyzes the oxidation of the substrate into acetaldehyde (Gorisch, 2003). Franden et al. (2018) has reported improved growth and EG utilization upon overexpression of Glyoxylate carboligase $(\mathrm{gcl})$ operon in the engineered P. putida KT2440 (ATCC 47054)

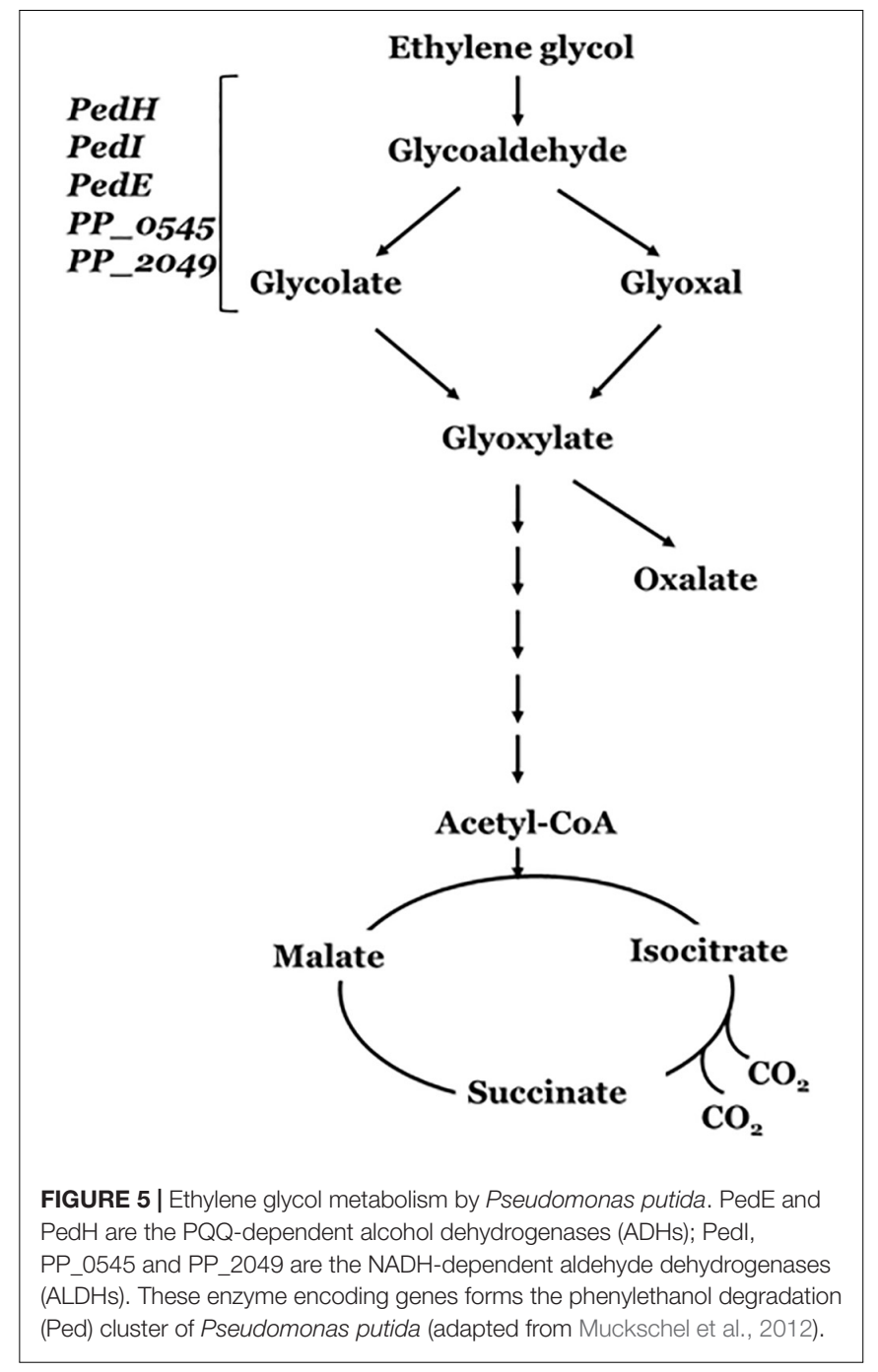

strain. In addition, the engineered strain enables conversion of EG to medium-chain-length polyhydroxyalkanoates (mcl-PHAs) (Mohanan et al., 2020).

\section{PETRO-PLASTIC WITH HETERO/CARBAMATE(URETHANE) BONDS: POLYURETHANE}

Polyurethanes (PUs) are widely used synthetic polymers finding applications as microplastics in the medical (e.g., catheters) and industrial products (especially as foams and domestic consumables; Figure 1). PUs also finds use in adhesives, insulation, coats, tires, sponges, paints, and fibers (Howard, 2002; Zheng et al., 2005; Shah et al., 2008). PU is not abundant in nature and only a few microbial strains have been reported to efficiently degrade it. Extensive degradation of plastic waste generates microplastic (particles with a size smaller than $5 \mathrm{~mm}$ ) (Zheng et al., 2005; Shah et al., 2008; do Sul and Costa, 2014). Microplastics pose a major problem to environment and human 


\section{Polyurethane}

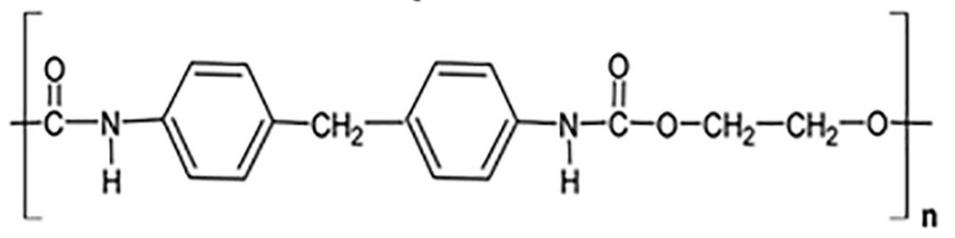

PU Esterase
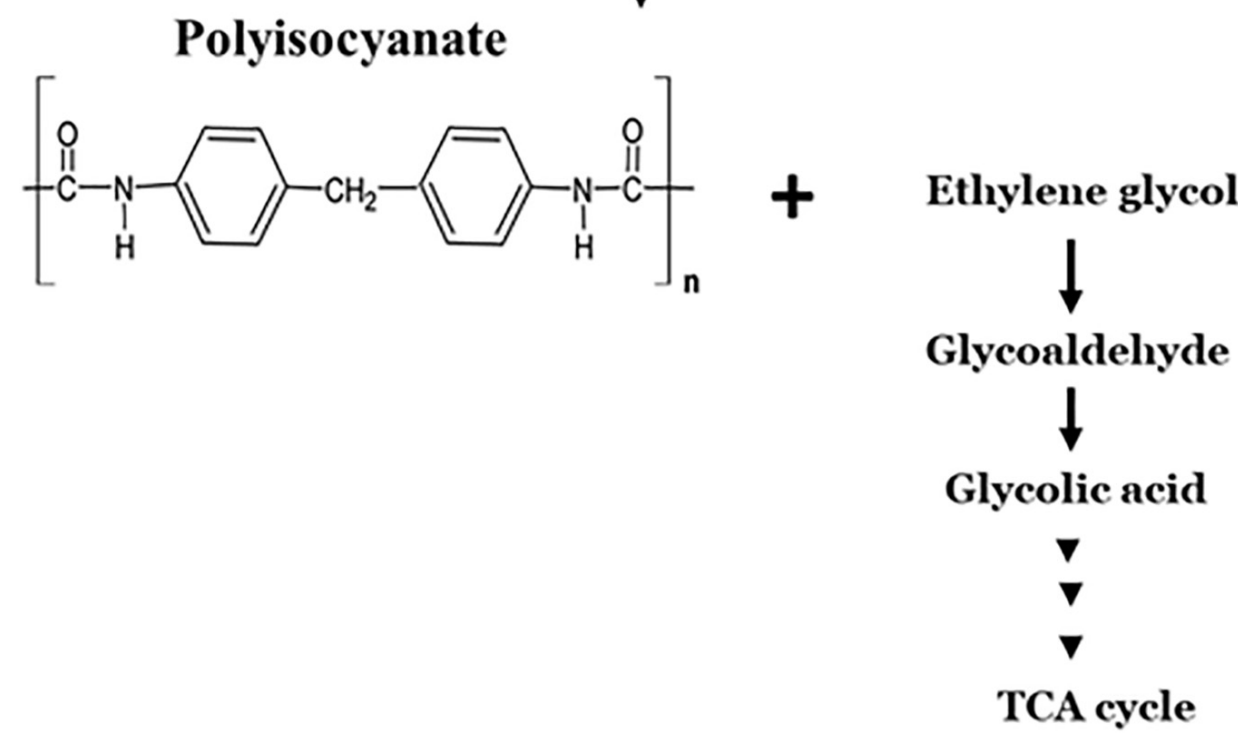

FIGURE 6 | Microbial degradation pathway of polyurethane.

health, since the particles are shown to attract and store toxic compounds such as polybrominated diphenyl ethers (PBDs), polychlorinated biphenyls (PCBs) and bisphenols (Bouwmeester et al., 2015). Microplastics comprising PU foams, PE particles, and PP particles, form the major pollutants with a half-life estimated to be $\sim 50$ years in sea water (Plastics Europe, 2017; Sharma and Chatterjee, 2017; Kim et al., 2015) and polluting coastal and marine habitats (Sharma and Chatterjee, 2017).

$\mathrm{PU}$ is biodegraded through hydrolytic cleavage of urethane bonds (Nakajima-Kambe et al., 1999; Figure 6). Few fungal and bacterial species are shown to degrade polyester-polyurethane through enzymatic hydrolysis of ester linkages (Nakajima-Kambe et al., 1999; Howard, 2002; Table 2). Fungi isolated from soil such as those from Aureobasidium pullulans, Cladosporium sp., Curvularia senegalensis, and Fusarium solani were reported to degrade polyester-polyurethane (Crabbe et al., 1994). A variety of bacterial strains were able to use polyester-polyurethane polymers as carbon, nitrogen and energy source for growth, e.g., P. aeruginosa (Kay et al., 1991), Corynebacterium sp. (Kay et al., 1991), Comamonas acidovorans (Nakajima-Kambe et al., 1995), Pseudomonas fluorescens (Howard and Blake, 1998), Acinetobacter calcoaceticus (El-Sayed et al., 1996), and Bacillus subtilis (Rowe and Howard, 2002).

\section{ENZYMES INVOLVED IN DEGRADATION OF SYNTHETIC POLYMERS}

Petro-pastics are well known to be highly recalcitrant to natural biodegradation processes. However, as discussed above, some microorganisms can degrade certain polymers, under certain conditions. Microorganisms that degrade plastic may be considered living bioreactors. Polymer chains are broken by enzymes secreted by the microbes, and the hydrolysis products are taken up and metabolized. The identities of enzymes involved in the degradation petro-polymers like PE and PP, and the mechanisms of these enzyme-substrate interactions, are largely unknown. However, some progress in identifying the types of enzymes involve in petro-polymer degradation has been made. Enzymatic activities include hydrolysis/oxidation/hydroxylation, resulting in cleavage of the polymer chains into oligomers and monomers. From the point of view of enzymatic degradation, petro-polymers can be classified in two groups; hydrolysable (PET and PUR) and non-hydrolysable (PE, PS, PP, and PVC). The biodegradation pathways utilized by these two groups are significantly different (Wei and Zimmermann, 2017). The ester bonds in petro-plastic polymers are the same as ester bonds in other polyester polymers, and enzymes that cleave ester bonds are 
known as esterases. There are many different types of esterase, which differ in their protein structure, substrate specificity, and biological functions. Polymers with hydrolysable ester bonds in their backbones, such as PET and PUR, are more susceptible to biodegradation than polymers with carbon chain backbones, like PE, PS, PP and PVC. Enzymes that degrade the high-molecular weight polymers of PET and ester-based PU are known and details of their activities have been characterized. However, to date, no specific enzymes with the ability to biodegrade PE, PS, $\mathrm{PP}$, or PVC have been well characterized, and the mechanisms of degradation the covalent bonds in carbon backbone petropolymers are currently unknown. Figure 7 shows the selected enzymes from different microorganisms capable of hydrolyzing petro-polymers and synthetic polyesters.

It is presumed that large, high molecular weight polymers are first degraded by extracellular enzymes, secreted by microorganisms, into smaller subunits (oligomers and/or dimers) that can be incorporated into the microbial cells. Once in the cells, these degradation products are channelled through the classical catabolic pathways to yield energy and reducing power for cell growth. The microbial degradation of petro-polymers is known as a very slow process (Table 1). The high resistance of these polymers mainly stems from their high molecular weight and the extremely hydrophobic surfaces, which prevent these molecules from transiting the cell wall. Different levels of degradability are thought to depend on the extent of amorphous and crystalline forms, and the presence of strong $\mathrm{C}-\mathrm{C}$ bonds, which are very resistant to attack by enzymes.

Cutinases (EC 3.1.1.74) are a sub-class of esterase enzymes that have gained importance because of their ability to hydrolyze polyesters with a high molar mass (Chen et al., 2013). Cutinases are $\alpha / \beta$ hydrolases or carboxylic ester hydrolases originally extracted from plant pathogenic fungi, e.g., Fusarium solani pisi (Purdy and Kolattukudy, 1975; Kollattukudy, 1981; Heredia, 2003). The enzyme caught attention for their phytopathogenicity as they can hydrolyze the cutin of the cuticular layer in leaves or the suberin in bark.

A polyesterase capable of hydrolyzing aromatic polyesters (primarily PET) was first reported from Thermobifida fusca by a German research group (Müller et al., 2005). A variety of cutinases were also purified and characterized from bacterial strains of thermophilic actinomycetes (Zimmermann and Billig, 2011; Chen et al., 2013; Wei et al., 2014a), for instance Thermomonospora fusca (Fett et al., 1999), Thermobifida fusca (Kleeberg et al., 2005), Thermobifida alba (Hu et al., 2010), Thermobifida cellulosilytica (Herrero Acero et al., 2011), and Thermomonospora curvata (Wei et al., 2014b; Islam et al., 2019). Cutinases have also been reported to hydrolyse aliphatic polyesters e.g., PCL (Kleeberg et al., 2005; Baker et al., 2012) as well as aliphatic aromatic co-polyesters such as PET (Herrero Acero et al., 2011; Ribitsch et al., 2012a; Roth et al., 2014; Wei et al., 2014b; Gamerith et al., 2017) and polytrimethylene terephthalate (PTT) (Eberl et al., 2009).

Several studies on PET degradation revealed that all known PET hydrolases belong to the cutinase group (Tokiwa and Calabia, 2007; Zimmermann and Billig, 2011; Baker et al., 2012). Further, crystal structures of PET-hydrolyzing cutinases have been analyzed for the substrate-binding domain and possible mutations to improve the degradation rates. Protein engineering studies on cutinases (Silva et al., 2011; Herrero Acero et al., 2013) and fusion to binding domains (Zhang et al., 2010; Ribitsch et al., 2013, 2015) have demonstrated enhanced polymer degradation. In latter approach, cutinases (from $T$. cellulosilytica and T. fusca) were fused to binding domains like polyhydroxyalkanoate binding modules (PBM) (Ribitsch et al., 2013), carbohydrate binding modules (CBM) (Zhang et al., 2010; Ribitsch et al., 2013) and hydrophobins (Ribitsch et al., 2015). In all cases, the activity and thus the hydrolysis toward PET was enhanced compared to the wild-type enzyme, which did not possess a hydrophobic binding-domain for a targeted polymer degradation. The fusion of hydrophobins (HBF4 or HBF7) to a PET-degrading cutinase (Thc_Cut1) enhanced the depolymerization up to 16 times (Ribitsch et al., 2015). Fusion of a PBM to the enzyme enhanced hydrolysis by 11-fold compared to the wild-type enzyme (Ribitsch et al., 2013).

Enzymatic hydrolysis of PET may occur via two mechanisms: (1) enzymatic surface modification of polyester fibers; or (2) enzymatic hydrolysis of the polymer. Enzymatic surface modification is carried out by PET surface-modifying enzymes or hydrolases, such as lipases, cutinases, carboxylesterases and proteases. These enzymes do not hydrolyze the PET polymer chains, but degrade the surface of the polymer. However, management of PET waste requires substantial degradation of the PET chains. Very few cutinases have been recognized as PET hydrolases that can degrade the inner block of PET (by at least 10\%) since the first discovery of PET hydrolase by Müller et al. (2005).

Several hydrolases have been tested for the surface hydrophilization of PET fibers, such as lipases from Candida antarctica (Vertommen et al., 2005), Triticum aestivum (Nechwatal et al., 2006), Thermomyces lanuginosus (Eberl et al., 2009), and Burkholderia spp. (Lee and Chung, 2009); fungal cutinases from Fusarium solani (Alisch-Mark et al., 2006; O’Neill et al., 2007), Penicillium citrinum (Liebminger et al., 2007), and Aspergillus oryzae, Humicola insolens (Ronqvist et al., 2009); and those from actinomycetes, Saccharomonospora viridis (Kawai et al., 2017), T. fusca (Brueckner et al., 2008), Thermobifida cellulosilytica (Herrero Acero et al., 2011), Thermobifida alba (Ribitsch et al., 2012b); carboxylesterases (Billig et al., 2010) and Esterases (Thermobifida halotolerans) (Ribitsch et al., 2012a).

Besides crystallinity, hydrophobicity, surface topology, and molecular size of synthetic polymers (Tokiwa et al., 2009; Webb et al., 2013; Wei and Zimmermann, 2017), enzymatic PET degradation is also dependent on the reaction temperatures ( $>$ Tg, preferably $65-70^{\circ} \mathrm{C}$ in aqueous solution), and the enzyme structure (active site accessibility to the polymer surface; Zumstein et al., 2017; Islam et al., 2019). Polyesterases preferentially hydrolyzes amorphous regions of PET (Welzel et al., 2002; Brueckner et al., 2008; Ronqvist et al., 2009; Donelli et al., 2010; Gamerith et al., 2017). Increased crystallinity limits the movement of polymer chains and therefore, decreases the availability of polymer chains for enzymatic attack. None of the PET hydrolases reported so far degrades the crystalline polymers such as those in PET bottles, textiles, and biaxially stretched 


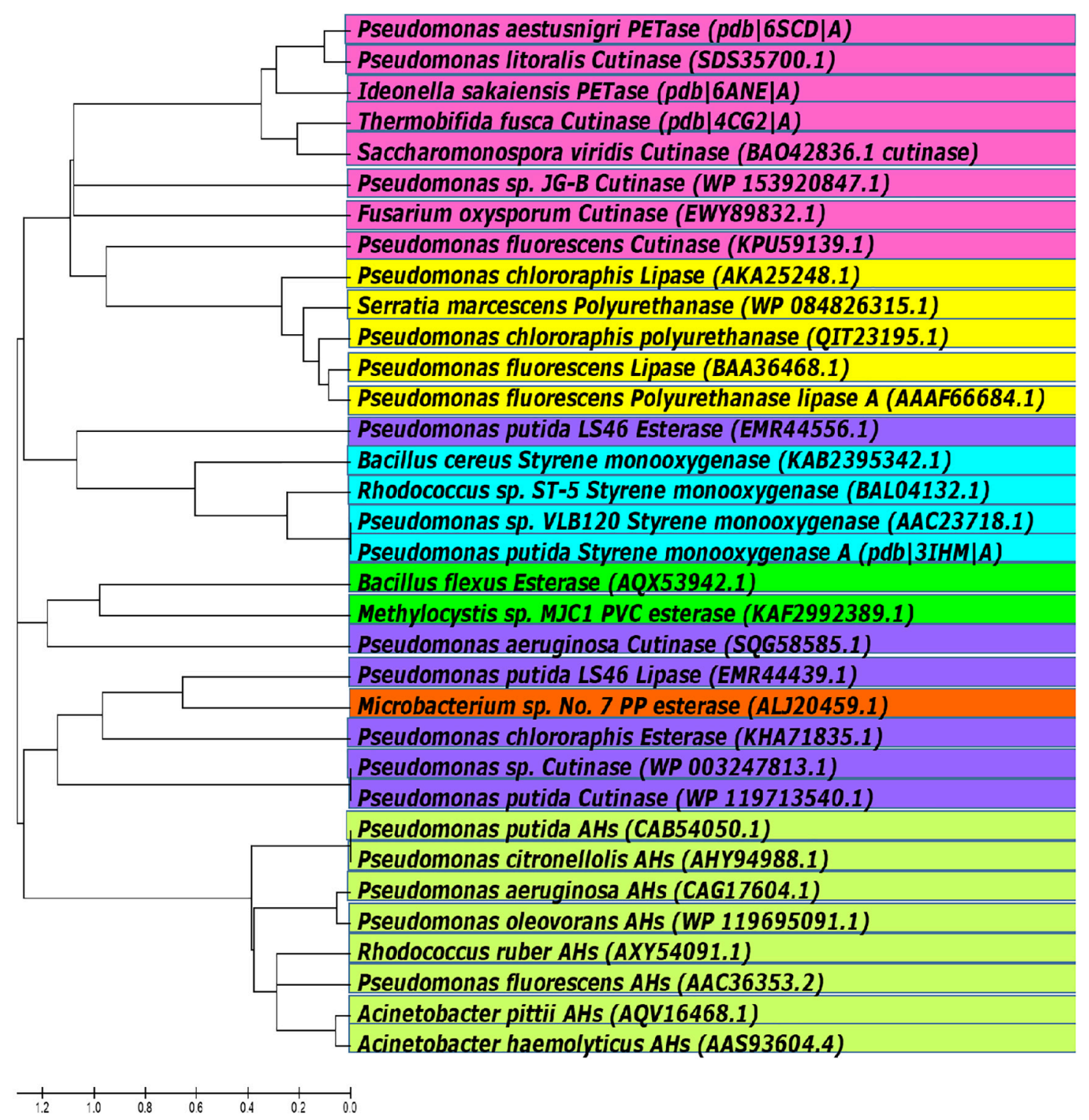

$\begin{array}{llll}\begin{array}{l}\text { Polyethylene terephthalate (PET) } \\ \text { polypropylene (PP) }\end{array} & \begin{array}{l}\text { Polyurethane (PU) } \\ \text { Polystyrene (PS) }\end{array} \\ & \begin{array}{l}\text { Polyethylene (PE) } \\ \text { Uncharacterized }\end{array}\end{array}$

FIGURE 7 | Phylogenetic relationships among bacteria, based on amino acid sequence homologies of petro-polymer degrading enzymes. Majority of the currently known and biochemically characterized, as well as uncharacterized enzymes, were included in the alignment. The tree was constructed with Molecular Evolutionary Genetics Analysis version 5 (MEGA5).

films. Degradation of the polyesters was found promoted at higher temperatures. Increased temperatures (over $55^{\circ} \mathrm{C}$ ) also contributed to the disinfection of digested plastic waste (Islam et al., 2019).

Polyhydroxyalkanoate binding modules (PBM) have also been used to degrade polyurethane polyester co-polymers (Gamerith et al., 2016). The fusion of polyamidase and PBM (PA-PBM) improved hydrolysis of PU polymers by fourfold relative to the native enzyme (Gamerith et al., 2016). Many studies were conducted on the esterase-activities in the microbial degradation of polyester-polyurethane (Akutsu et al., 1998; Rowe and Howard, 2002). Membrane associated (e.g., PudA) (Akutsu et al., 1998) and extracellular (e.g., PueA, PueB) (Allen et al., 1999; Ruiz et al., 1999; Vega et al., 1999). Polyurethanases (PUases) 
from Pseudomonas chlororaphis, P. fluorescens, and Comamonas acidovorans TB-35) were characterized. The degradation of polyurethane, like other polymers, occurs in a two-step process with PU-esterase (PudA). Firstly, a surface-binding domain promotes hydrophobic adsorption of PudA to the PU surface and, hydrolysis of ester bonds forms the second step (Akutsu et al., 1998).

Islam et al. (2019) reported an improved binding and depolymerization of polyester-polyurethane nanoparticles by fusion of the anchor peptide, Tachystatin A2 (TA2) to the cutinase from Thermomonospora curvata (Tcur1278). Anchor peptides are metal-binding adhesion peptides, which provide a versatile tool for a targeted degradation of microplastics and/or polymers such as PET, PP, and PU, at ambient temperature in highly diluted suspensions. Tachystatin A2 (44 aa) has been known to from a dense monolayer on PP and PS surfaces (Osaki et al., 1999). TA2 was reengineered via a directed evolution campaign (following the KnowVolution strategy) (Rübsam et al., 2018b) and employing a specialized diversity generation protocol for short peptides (termed PePevo) to create variants with increased binding to PS and PP in the presence of surfactants (LAS and Triton X-100) (Rübsam et al., 2018a). The fusion of TA2 to the cutinase (Tcur1278) accelerated the degradation of polyesterpolyurethane nanoparticles by a factor of 6.6 relative to the wild-type Tcur1278.

Blending of the plastics with certain types of natural polymers, such as starch, has been shown to increase their biodegradation (Otake et al., 1995; Zheng et al., 2005; Ammala et al., 2011; Karimi and Biria, 2019). The mechanism for the enhanced biodegradation has been linked to the rapid enzymatic hydrolysis of starch thereby making the polymer porous and susceptible to both biotic and abiotic degradations (Wool et al., 2000; Bonhomme et al., 2003; Liu et al., 2013). Karimi and Biria (2019) reported the biodegradation of LDPE-starch blend samples by an alpha-amylase aqueous solution. Amylase was found to have a co-metabolic behavior and hydrolyzed the primary specific substrate (starch) as well as PE molecules. Moreover, the addition of hydrophilic starch into hydrophobic PE was found to enhance the biodegradability of both polymers (Hoque et al., 2013).

The AHs of Pseudomonas sp. strain E4 were shown to play an important role in biodegradation of a non-oxidized LMWPE (Yoon et al., 2012). LMWPE has a molecular weight that was well above the upper-limit that can penetrate microbial membranes. Jeon and Kim (2016) reported the functional characteristics of alkane monooxygenases (AlkB1 and AlkB2) from $P$. aeruginosa involved in LMWPE biodegradation and found that the regulation mechanism of these enzymes was different, and that the AlkB2 enzyme was more effective in degrading LMWPE than the AlkB1 enzyme.

Danso et al. (2018) used Markov Model-based search strategy to show that a surprisingly large variety of potential polyesterases still needs to be discovered particularly in bacteria, which are currently not considered as a prime source for cutinases (Danso et al., 2018). The predominantly marine Pseudomonas lineage, which includes halophilic, psychrophilic, hydrocarbonoclastic, and heavy metal-tolerant species, is one such example; these were found as a source for PU hydrolyzing enzymes (Wilkes and
Aristilde, 2017), while the Guebitz-group at the Austrian Centre of Industrial Biotechnology, Austria revealed the polyesterase activity in Pseudomonads (Haernvall et al., 2017; Wallace et al., 2017). Polyesterase genes in Pseudomonas pertucinogena have been confirmed by sequence homology searches (Bollinger et al., 2018). Further, many strains of Pseudomonas have been reported to degrade a wide-range of recalcitrant compounds and/or plastics such as PE, PS, PP, and PVC (Giacomucci et al., 2019).

\section{CONCLUSION}

Biodegradation of petroleum-derived polymers has been an innovative area of research focused on solving plastic pollution in the environment. This review has discussed the microorganisms and enzymes reported to biodegrade these synthetic polymers. Many strains of Pseudomonas and Bacillus have been observed to degrade complex, recalcitrant compounds such as polyaromatic hydrocarbons, and have been associated with the partial degradation of a wide-range of petro-plastics, including PE, PS, PP, PVC, PET and ester-based PU. The gut microbes in insects have also been found to depolymerize PE, PS and PVC polymers. Enzymes specifically associated with depolymerization of PET and ester-based PU have been identified and intensively studied, while enzymes that effectively depolymerize PE, PP, PS, and PVC have not yet been identified and characterized. Further analyses of the genes and/or gene products (enzymes) that hydrolyze the high molecular weight petro-plastic polymers may lead to greater understanding of the underlying molecular mechanisms of biodegradation. Research focusing on digestive enzyme(s) in plastic-degrading invertebrates and their gut microbes could also lead to novel approach for plastic degradation, especially for persistent non-hydrolyzable polymers. Based on these knowledge, genetic engineering approaches to create recombinant microbial strains and/or enzymes could be adopted as the preferred strategy to enhance biodegradation of the synthetic petroleum based plastic waste.

\section{AUTHOR CONTRIBUTIONS}

NM was the primary author of the manuscript. ZM and DL contributed to certain sections of the manuscript, as well as overall editing and proof-reading. PS contributed to training of $\mathrm{NM}$ and ZM. All authors contributed to the article and approved the submitted version.

\section{FUNDING}

This work was supported by the Natural Sciences and Engineering Research Council (NSERC) of Canada through a Discovery Grant (RGPIN-04945-2017) held by DL. NSERC Discovery grants are operating grants that enable academic researcher to pursue lead-edge research and include a small allocation of funds of publication of research results in international, peer-reviewed journals. 


\section{REFERENCES}

Abrusci, C., Pablos, J. L., Marín, I., Espí, E., Corrales, T., and Catalina, F. (2013). Comparative effect of metal stearates as pro-oxidant additives on bacterial biodegradation of thermal- and photo-degraded low density polyethylene mulching films. Int. Biodeterior. Biodegrad. 83, 25-32. doi: 10.1016/j.ibiod. 2013.04.002

Ahmed, T., Shahid, M., Azeem, F., Rasul, I., Shah, A. A., Noman, M., et al. (2018). Biodegradation of plastics: current scenario and future prospects for environmental safety. Environ. Sci. Pollut. Res. Int. 25, 7287-7298. doi: 10.1007/ s11356-018-1234-9

Akutsu, Y., Nakajima-Kambe, T., Nomura, N., and Nakahara, T. (1998). Purification and properties of a polyester polyurethane-degrading enzyme from Comamonas acidovorans TB-35. Appl. Environ. Microbiol. 64, 62-67. doi: 10. 1128/AEM.64.1.62-67.1998

Albertsson, A. C., Anderson, S. O., and Karlsson, S. (1987). Mechanism of biodegradation of polyethylene. Polym. Degrad. Stab. 18, 73-87. doi: 10.1016/ 0141-3910(87)90084-X

Albertsson, A. C., and Karlsson, S. (1990). The influence of biotic and abiotic environments on the degradation of polyethylene. Prog. Polym. Sci. 15, 177-192. doi: 10.1016/0079-6700(90)90027-X

Albertsson, A. C., and Karlsson, S. (1993). Aspects of biodeterioration of inert and degradable polymers. Int. Biodeterior. Biodegrad. 31, 161-170. doi: 10.1016/ 0964-8305(93)90002-J

Ali, M. I., Ahmed, S., Javed, I., Ali, N., Atiq, N., Hameed, A., et al. (2014). Biodegradation of starch blended polyvinyl chloride films by isolated Phanerochaete chrysosporium PV1. Int. J. Environ. Sci. Tech. 11, 339-348. doi: 10.1007/s13762-013-0220-5

Alisch-Mark, M., Herrmann, A., and Zimmermann, W. (2006). Increase of the hydrophilicity of polyethylene terephthalate fibers by hydrolases from Thermomonospora fusca and Fusarium solani f. sp. pisi. Biotechnol. Lett. 28, 681-685. doi: 10.1007/s10529-006-9041-7

Allen, A. B., Hilliard, N. P., and Howard, G. T. (1999). Purification and characterization of a soluble polyurethane degrading enzyme from Comamonas acidovorans. Int. Biodeterior. Biodegrad. 43, 37-41. doi: 10.1016/S09648305(98)00066-3

Alvarez, H. M. (2003). Relationship between $\beta$-oxidation pathway and the hydrocarbon-degrading profile in actinomycetes bacteria. Int. Biodeterior. Biodegrad. 52, 35-42. doi: 10.1016/S0964-8305(02)00120-8

Álvarez-Hernández, C., Cairós, C., López-Darias, J., Mazzetti, E., HernándezSánchez, C., González-Sálamo, J., et al. (2019). Microplastic debris in beaches of Tenerife (Canary Islands, Spain). Mar. Pollut. Bull. 146, 26-32. doi: 10.1016/ j.marpolbul.2019.05.064

Ammala, A., Bateman, S., Deana, K., Petinakis, E., Sangwan, P., Wong, S., et al. (2011). An overview of degradable and biodegradable polyolefins. Prog. Polym. Sci. 36, 1015-1049. doi: 10.1016/j.progpolymsci.2010.12.002

Andrady, A. L. (2011). Microplastics in the marine environment. Mar. Pollut. Bull. 62, 1596-1605. doi: 10.1016/j.marpolbul.2011.05.030

Arias, S., Olivera, E. R., Arcos, M., Naharro, G., and Luengo, J. M. (2008). Genetic analyses and molecular characterization of the pathways involved in the conversion of 2-phenylethylamine and 2-phenylethanol into phenylacetic acid in Pseudomonas putida U. Environ. Microbiol. 10, 413-432. doi: 10.1111/j. 1462-2920.2007.01464.x

Arkatkar, A., Juwarkar, A. A., Bhaduri, S., Uppara, P. V., and Doble, M. (2010). Growth of Pseudomonas and Bacillus biofilms on pretreated polypropylene surface. Int. Biodeterior. Biodegrad. 64, 530-536. doi: 10.1016/j.ibiod.2010.06. 002

Austin, H. P., Allen, M. D., Donohoe, B. S., Rorrer, N. A., Kearns, F. L., Silveira, R. L., et al. (2018). Characterization and engineering of a plastic degrading aromatic polyesterase. Proc. Natl. Acad. Sci. U.S.A. 115, E4350-E4357. doi: 10.1073/pnas. 1718804115

Auta, H. S., Emenike, C. U., and Fauziah, S. H. (2017). Screening for Polypropylene degradation potential of bacteria isolated from mangrove ecosystems in Penninsular Malaysia. IJBBB 7, 245-251. doi: 10.17706/ijbbb.2017.7.4. 245-251

Baker, M. A.-M., and Mead, J. (2002). "Thermoplastics," in Handbook of Plastics, Elastomers and Composites, 4th Edn, ed. C. A. Harper (New York, NY: McGrawHill), 1-90.
Baker, P. J., Poultney, C., Liu, Z., Gross, R. A., and Montclare, J. K. (2012). Identification and comparison of cutinases for synthetic polyester degradation. Appl. Microbiol. Biotechnol. 93, 229-240. doi: 10.1007/s00253-011-3402-4

Barnes, D. K. A., Galgani, F., Thompson, R. C., and Barlaz, M. (2009). Accumulation and fragmentation of plastic debris in global environments. Phil. Trans. R. Soc. B 364, 1985-1998. doi: 10.1098/rstb.2008.0205

Bergmann, M., Mützel, S., Primpke, S., Tekman, M. B., Trachsel, J., and Gerdts, G. (2019). White and wonderful? Microplastics prevail in snow from the Alps to the Arctic. Sci. Adv. 5:eaax1157. doi: 10.1126/sciadv.aax1157

Bhatia, S. K., Gurav, R., Choi, T. R., Jung, H. R., Yang, S. Y., Moon, Y. M., et al. (2019a). Bioconversion of plant biomass hydrolysate into bioplastic (polyhydroxyalkanoates) using Ralstonia eutropha 5119. Bioresour. Technol. 271, 306-315. doi: 10.1016/j.biortech.2018.09.122

Bhatia, S. K., Gurav, R., Choi, T. R., Jung, H. R., Yang, S. Y., Song, H. S., et al. (2019b). Poly(3-hydroxybutyrate-co-3-hydroxyhexanoate) production from engineered Ralstonia eutropha using synthetic and anaerobically digested food waste derived volatile fatty acids. Int. J. Biol. Macromol. 133, 1-10. doi: 10.1016/j.ijbiomac.2019.04.083

Billen, P., Khalifa, L., Van Gerven, F., Tavernier, S., and Spatari, S. (2020). Technological application potential of polyethylene and polystyrene biodegradation by macro-organisms such as mealworms and wax moth larvae. Sci. Total Environ. 735:139521. doi: 10.1016/j.scitotenv.2020.139521

Billig, S., Oeser, T., Birkemeyer, C., and Zimmermann, W. (2010). Hydrolysis of cyclic poly(ethylene terephthalate) trimers by a carboxylesterase from Thermobifida fusca KW3. Appl. Microbiol. Biotechnol. 87, 1753-1764. doi: 10.1007/s00253-010-2635-y

Blank, L. M., Ionidis, G., Ebert, B. E., Bühler, B., and Schmid, A. (2008). Metabolic response of Pseudomonas putida during redox biocatalysis in the presence of a second octanol phase. FEBS J. 275, 5173-5190. doi: 10.1111/j.1742-4658.2008. 06648.x

Bollinger, A., Thies, S., Katzke, N., and Jaeger, K. E. (2018). The biotechnological potential of marine bacteria in the novel lineage of Pseudomonas pertucinogena. Microb. Biotechnol. 13, 19-31. doi: 10.1111/1751-7915.13288

Bombelli, P., Howe, C. J., and Bertocchini, F. (2017). Polyethylene bio-degradation by caterpillars of the wax moth Galleria mellonella. Curr. Biol. 27, 292-293. doi: 10.1016/j.cub.2017.02.060

Bonhomme, S., Cuer, A., Delort, A. M., Lemaire, J., Sancelme, M., and Scott, G. (2003). Environmental biodegradation of polyethylene. Polym. Degrad. Stab. 81, 441-452. doi: 10.1016/S0141-3910(03)00129-0

Bouwmeester, H., Hollman, P. C., and Peters, R. J. (2015). Potential health impact of environmentally released micro-and nanoplastics in the human food production chain: experiences from nanotoxicology. Environ. Sci. Technol. 49, 8932-8947. doi: 10.1021/acs.est.5b01090

Brandon, A. M., Gao, S. H., Tian, R., Ning, D., Yang, S. S., Zhou, J., et al. (2018). Biodegradation of polyethylene and plastic mixtures in mealworms (Larvae of Tenebrio molitor) and effects on the gut microbiome. Environ. Sci.Technol. 52, 6526-6533. doi: 10.1021/acs.est.8b02301

Braun, D. (1975). "Recent progress in the thermal and photochemical degradation of poly(vinyl chloride)," in Degradation and Stabilization of Polymers, ed. Geuskens (London: Wiley), 23-41.

Braun, D. (2004). Poly (vinyl chloride) on the way from the 19th century to the 21st century. J. Polym. Sci. Part A Polym. Chem. 42, 578-586. doi: 10.1002/pola. 10906

Browne, M. A., Crump, P., Niven, S. J., Teuten, E., Tonkin, A., Galloway, T., et al. (2011). Accumulation of microplastic on shorelines worldwide: sources and sinks. Environ. Sci. Technol. 45, 9175-9179. doi: 10.1021/es201811s

Brueckner, T., Eberl, A., Heumann, S., Rabe, M., and Guebitz, G. M. (2008). Enzymatic and chemical hydrolysis of poly(ethylene terephthalate) fabrics. J. Polym. Sci. Pat A Polym. Chem. 46, 6435-6443. doi: 10.1002/pola.22952

Bumpus, J. A., and Aust, S. D. (1987). "Mineralization of recalcitrant environmental pollutants by the white rot fungus," in Proceedings of The National Conference on Hazardous Wastes and Hazardous Materials Washington, DC, 146-151.

Bumpus, J. A., Fernando, T., Mileski, G. J., and Aust, S. D. (1988). "Biological oxidation of organic compounds by enzymes from a white rot fungus," in Proceedings of The 14th Annual Research Symposium Oak Ridge, TN, 1-16.

Cacciari, P., Quatrini, G., Zirletta, E., Mincione, V., Vinciguerra, P., Lupattelli, P., et al. (1993). Isotactic polypropylene biodegradation by a microbial community: 
physicochemical characterization of metabolites produced. Appl. Environ. Microbiol. 59, 3695-3700. doi: 10.1128/AEM.59.11.3695-3700.1993

Carpenter, E. J., and Smith, K. L. Jr. (1972). Plastics on the Sargasso Sea surface. Science 175, 1240-1241. doi: 10.1126/science.175.4027.1240

Caskey, W. H., and Taber, W. A. (1981). Oxidation of ethylene glycol by a salt requiring bacterium. Appl. Environ. Microbiol. 42, 180-183. doi: 10.1128/AEM. 42.1.180-183.1981

Cassone, B. J., Grove, H. C., Elebute, O., Villanueva, S. M. P., and LeMoine, C. M. R. (2020). Role of the intestinal microbiome in low-density polyethylene degradation by caterpillar larvae of the greater wax moth, Galleria mellonella. Proc. R. Soc. B 287:20200112. doi: 10.1098/rspb.2020.0112

Chen, S., Su, L., Chen, J., and Wu, J. (2013). Cutinase: characteristics, preparation and application. Biotechnol. Adv. 31, 1754-1767. doi: 10.1016/j.biotechadv. 2013.09.005

Child, J., and Willetts, A. (1978). Microbial metabolism of aliphatic glycols. Bacterial metabolism of ethylene glycol. Biochim. Biophys. Acta 538, 316-327. doi: 10.1016/0304-4165(78)90359-8

Crabbe, J. R., Campbell, J. R., Thompson, L., Walz, S. L., and Schultz, W. W. (1994). Biodegradation of a colloidal ester-based polyurethane by soil fungi. Int Biodeterior. Biodegrad. 33, 103-113. doi: 10.1016/0964-8305(94)90030-2

Danso, D., Schmeisser, C., Chow, J., Zimmermann, W., Wei, R., Leggewie, C., et al. (2018). New insights into the function and global distribution of polyethylene terephthalate (PET)-degrading bacteria and enzymes in marine and terrestrial metagenomes. Appl. Environ. Microbiol. 84:e02773-17. doi: 10.1128/AEM. 02773-17

Das, G., Bordoloi, N. K., Rai, S. K., Mukherjee, A. K., and Karak, N. (2012). Biodegradable and biocompatible epoxidized vegetable oil modified thermostable poly(vinyl chloride): thermal and performance characteristics post biodegradation with Pseudomonas aeruginosa and Achromobacter sp. J. Hazard Mater. 209-210, 434-442. doi: 10.1016/j.jhazmat.2012.01.043

Decker, C. (1984). "Photodegratadion of PVC," in Degradation and Stabilization of PVC, ed. Owen (London: Elsevier Applied Science Publication), 81-136. doi: 10.1007/978-94-009-5618-6_3

DeLey, J., and Kersters, K. (1964). Oxidation of aliphatic glycols by acetic acid bacteria. Bacteriol. Rev. 28, 164-180. doi: 10.1128/MMBR.28.2.164-180.1964

Di Gennaro, P., Colmegna, A., Galli, E., Sello, G., Pelizzoni, F., and Bestetti, G. (1999). A new biocatalyst for production of optically pure aryl ep-oxides by styrene monooxygenase from Pseudomonas fluorescens ST. Appl. Environ. Microbiol. 65, 2794-2797. doi: 10.1128/AEM.65.6.2794-2797.1999

do Sul, J. A. I., and Costa, M. F. (2014). The present and future of microplastic pollution in the marine environment. Environ. Pollut. 185, 352-364. doi: 10. 1016/j.envpol.2013.10.036

Donelli, I., Freddi, G., Nierstrasz, V. A., and Taddei, P. (2010). Surface structure and properties of poly(ethylene terephthalate) hydrolyzed by alkali and cutinase. Polym. Degrad. Stab. 95, 1542-1550. doi: 10.1016/j.polymdegradstab.2010.06. 011

Eberl, A., Heumann, S., Brueckner, T., Araujo, R., Cavaco-Paulo, A., Kaufmann, F., et al. (2009). Enzymatic surface hydrolysis of poly(ethylene terephthalate) and bis(benzoyloxyethyl)terephthalate by lipase and cutinase in the presence of surface active molecules. J. Biotechnol. 143, 207-212. doi: 10.1016/j.jbiotec. 2009.07.008

El-Sayed, A. H. M. M., Mahmoud, W. M., Davis, E. M., and Coughlin, R. W. (1996). Biodegradation of polyurethane coatings by hydrocarbon-degrading bacteria. Int. Biodeterior. Biodegrad. 37, 69-79. doi: 10.1016/0964-8305(95)00091-7

Eyheraguibel, B., Traikia, M., Fontanella, S., Sancelme, M., Bonhomme, S., Fromageot, D., et al. (2017). Characterization of oxidized oligomers from polyethylene films by mass spectrometry and NMR spectroscopy before and after biodegradation by a Rhodococcus rhodochrous strain. Chemosphere 184, 366-374. doi: 10.1016/j.chemosphere.2017.05.137

Fett, W. F., Wijey, C., Moreau, R. A., and Osman, S. F. (1999). Production of cutinase by Thermomonospora fusca ATCC 27730. J. Appl. Microbiol. 86, 561-568. doi: 10.1046/j.1365-2672.1999.00690.x

Fontanella, S., Bonhomme, S., Koutny, M., Husarova, L., Brusso, J. M., Courdavault, J. P., et al. (2010). Comparison of the biodegradability of various polyethylene films containing pro-oxidant additives. Polym. Degrad. Stab. 95, 1011-1021. doi: 10.1016/j.polymdegradstab.2010.03.009

Franden, M. A., Jayakody, L. N., Li, W.-J., Wagner, N. J., Cleveland, N. S., Michener, W. E., et al. (2018). Engineering Pseudomonas putida KT2440 for efficient ethylene glycol utilization. Metab. Eng. 48, 197-207. doi: 10.1016/j.ymben.2018. 06.003

Gajendiran, A., Krishnamoorthy, S., and Abraham, J. (2016). Microbial degradation of low-density polyethylene (LDPE) by Aspergillus clavatus strain JASK1 isolated from landfill soil. 3 Biotech. 6:52. doi: 10.1007/s13205-0160394-x

Gamerith, C., Herrero Acero, E., Pellis, A., Ortner, A., Vielnascher, R., Luschnig, D., et al. (2016). Improved enzymatic polyurethane hydrolysis by tuning enzymesorption. Polym. Degrad. Stab. 132, 69-77. doi: 10.1016/j. polymdegradstab.2016.02.025

Gamerith, C., Zartl, B., Pellis, A., Guillamot, F., Marty, A., Herrero Acero, E., et al. (2017). Enzymatic recovery of polyester building blocks from polymer blends. Process Biochem. 59, 58-64. doi: 10.1016/j.procbio.2017.01.004

Geyer, R., Jambeck, J. R., and Law, K. L. (2017). Production, use, and fate of all plastics ever made. Sci. Adv. 3:e1700782. doi: 10.1126/sciadv.1700782

Giacomucci, L., Raddadi, N., Soccio, M., Lotti, N., and Fava, F. (2019). Polyvinyl chloride biodegradation by Pseudomonas citronellolis and Bacillus flexus. New Biotechnol. 52, 35-41. doi: 10.1016/j.nbt.2019.04.005

Gilan, I., Hadar, Y., and Sivan, A. (2004). Colonization, biofilm formation and biodegradation of polyethylene by a strain of Rhodococcus ruber. Appl. Microbiol. Biotechnol. 65, 97-104. doi: 10.1007/s00253-004-1584-8

Goldman, A. S. (2010). ChemInform abstract: organometallic chemistry: carboncarbon bonds get a break. Nature 463, 435-436. doi: 10.1038/463435a

Gonzalez, C. F., Taber, W. A., and Zeitoun, M. A. (1972). Biodegradation of ethylene glycol by a salt-requiring bacterium. Appl. Microbiol. 24, 911-919. doi: 10.1128/AEM.24.6.911-919.1972

Gorghiu, L. M., Jipa, S., Zaharescu, T., Setnescu, R., and Mihalcea, I. (2004). The effect of metals on thermal degradation of polyethylenes. Polym. Degrad. Stab. 84, 7-11. doi: 10.1016/S0141-3910(03)00265-9

Gorisch, H. (2003). The ethanol oxidation system and its regulation in Pseudomonas aeruginosa. Biochim. Biophys. Acta 1647, 98-102. doi: 10.1016/ S1570-9639(03)00066-9

Grima, S., Bellon-Maurel, V., Feuilloley, P., and Silvestre, F. (2000). Aerobic biodegradation of polymers in solid-state conditions: a review of environmental and physicochemical parameter settings in laboratory simulations. J. Polym. Environ. 8, 183-195. doi: 10.1023/A:1015297727244

Guzik, M. W., Kenny, S. T., Duane, G. F., Casey, E., Woods, T., Babu, R. P., et al. (2014). Conversion of postconsumer polyethylene to the biodegradable polymer polyhydroxyalkanoate. Appl Microbiol Biotech. 98, 4223-4232. doi: 10.1007/s00253-013-5489-2

Haernvall, K., Zitzenbacher, S., Wallig, K., Yamamoto, M., Schick, M. B., Ribitsch, D., et al. (2017). Hydrolysis of ionic phthalic acid based polyesters by wastewater microorganisms and their enzymes. Environ. Sci. Technol. 51, 4596-4605. doi: 10.1021/acs.est.7b00062

Haines, J. R., and Alexander, M. (1974). Microbial degradation of high-molecularweight alkanes. Appl. Microbiol. 28, 1084-1085. doi: 10.1128/AEM.28.6.10841085.1974

Harada, T., and Hirabayashi, T. (1968). Utilization of alcohols by Hansenula miso. Agric. Biol. Chem. 32, 1175-1180. doi: 10.1271/bbb1961.32.1175

Harrison, J. P., Boardman, C., O'Callaghan, K., Delort, A. M., and Song, J. (2018). Biodegradability standards for carrier bags and plastic films in aquatic environments: a critical review. R. Soc. Open Sci. 5:171792. doi: 10.1098/rsos. 171792

Hasan, F., Shah, A. A., Hameed, A., and Ahmed, S. (2007). Synergistic effect of photo and chemical treatment on the rate of biodegradation of low density polyethylene by Fusarium sp. AF4. J. Appl. Polym. Sci. 105, 1466-1470. doi: 10.1002/app.26328

Heredia, A. (2003). Biochemical and biophysical characteristics of cutin, a plant barrier biopolymer. Biochim. Biophys. Acta 1620, 1-7. doi: 10.1016/S03044165(02)00510-X

Herrero Acero, E., Ribitsch, D., Steinkelner, G. T., Gruber, K., Greimel, K., Eiteljoerg, I., et al. (2011). Enzymatic surface hydrolysis of PET: effect of structural diversity on kinetic properties of cutinases from Thermobifida. Macromolecules 44, 4632-4640. doi: 10.1021/ma200949p

Herrero Acero, E., Ribitsch, D., Dellacher, A., Zitzenbacher, S., Marold, A., Steinkellner, G., et al. (2013). Surface engineering of a cutinase from Thermobifida cellulosilytica for improved polyester hydrolysis. Biotechnol. Bioeng. 110, 2581-2590. doi: 10.1002/bit.24930 
Hidalgo-Ruz, V., Gutow, L., Thompson, R. C., and Thiel, M. (2012). Microplastics in the marine environment: a review of the methods used for identification and quantification. Environ. Sci. Technol. 46, 3060-3075. doi: 10.1021/es203 1505

Ho, B. T., Roberts, T. K., and Lucas, S. (2018). An overview on biodegradation of polystyrene and modified polystyrene: the microbial approach. Crit. Rev. Biotechnol. 38, 1-13. doi: 10.1080/07388551.2017.1355293

Hoque, M. E., Ye, T. J., Yong, L. C., and Mohd Dahlan, K. Z. (2013). Sago starch-mixed low-density polyethylene biodegradable polymer: synthesis and characterization. J. Mater. 2013:365380. doi: 10.1155/2013/365380

Howard, G. T. (2002). Biodegradation of polyurethane: a review. Int. Biodeterior. Biodegrad. 49, 245-252. doi: 10.1016/S0964-8305(02)00051-3

Howard, G. T., and Blake, R. C. (1998). Growth of Pseudomonas fluorescens on a polyester-polyurethane and the purification and characterization of a polyurethanase-protease enzyme. Int. Biodeterior. Biodegrad. 42, 213-220. doi: 10.1016/S0964-8305(98)00051-1

Hu, X., Thumarat, U., Zhang, X., Tang, M., and Kawai, F. (2010). Diversity of polyester-degrading bacteria in compost and molecular analysis of a thermoactive esterase from Thermobifida alba AHK119. Appl. Microbiol. Biotechnol. 87, 771-779. doi: 10.1007/s00253-010-2555-x

Huang, C.-Y., Roan, M.-L., Kuo, M.-C., and Lu, W.-L. (2005). Effect of compatibiliser on the biodegradation and mechanical properties of highcontent starch/low-density polyethylene blends. Polym. Degrad. Stab. 90, 95105. doi: 10.1016/j.polymdegradstab.2005.02.015

Islam, S., Apitius, L., Jakob, F., and Schwaneberg, U. (2019). Targeting microplastic particles in the void of diluted suspensions. Environ. Int. 123, 428-435. doi: 10.1016/j.envint.2018.12.029

Iwamoto, A., and Tokiwa, Y. (1994). Enzymatic degradation of plastics containing polycaprolactone. Polym. Degrad. Stab. 45, 205-213. doi: 10.1016/01413910(94)90138-4

Jain, K., Bhunia, H., and Sudhakara Reddy, M. (2018). Degradation of polypropylene-poly L lactide blend by bacteria isolated from compost. Bioremed. J. 22, 3-4. doi: 10.1080/10889868.2018.1516620

Jasso, C. F., Gonzalez-Ortiz, L. J., Contrews, J. R., Mendizabal, M. E., and Mora, G. J. (1998). The degradation of high impact polystyrene with and without starch in concentrated activated sludge. Polym. Eng. Sci. 38, 863-869. doi: 10.1002/pen. 10252

Jeon, H. J., and Kim, M. N. (2015). Functional analysis of alkane hydroxylase system derived from Pseudomonas aeruginosa E7 for low molecular weight polyethylene biodegradation. Int. Biodeterior. Biodegrad. 103, 141-146. doi: 10.1016/j.ibiod.2015.04.024

Jeon, H. J., and Kim, M. N. (2016). Comparison of the functional characterization between alkane monooxygenases for low-molecular-weight polyethylene biodegradation. Int. Biodeterior. Biodegrad. 114, 202-208. doi: 10.1016/j.ibiod. 2016.06.012

Jumaah, O. S. (2017). Screening of plastic degrading bacteria from dumped soil area. IOSR J. Environ. Sci. Toxicol. Food Technol. 11, 93-98. doi: 10.9790/24021105029398

Kaczmarek, H., and Bajer, K. (2007). Biodegradation of plasticized poly(vinyl chloride) containing cellulose. J. Polym. Sci. Part B Polym. Phys. 45, 903-918. doi: $10.1002 /$ polb. 21100

Kaczmarek, H., Oldak, D., Malanowski, P., and Chaberska, H. (2005). Effect of short wavelength UV-irradiation on ageing of polypropylene/cellulose compositions. Polym. Degrad. Stab. 88, 189-198. doi: 10.1016/j.polymde gradstab.2004.04.017

Kaplan, D. L., Roy, H., and Jim, S. (1979). Biodegradation of polystyrene, poly(methyl methacrylate), and phenol formaldehyde. Appl. Environ. Microbiol. 38, 551-553. doi: 10.1128/AEM.38.3.551-553.1979

Karimi, M., and Biria, D. (2019). The promiscuous activity of alpha-amylase in biodegradation of low density polyethylene in a polymer-starch blend. Nat. Sci. Rep. 9:2612. doi: 10.1038/s41598-019-39366-0

Kataoka, M., Sasaki, M., Hidalgo, A. R., Nakano, M., and Shimizu, S. (2001). Glycolic acid production using ethylene glycol-oxidizing microorganisms. Biosci. Biotechnol. Biochem. 65, 2265-2270. doi: 10.1271/bbb.65.2265

Kawai, F., Kawase, T., Shiono, T., Urakawa, H., Sukigara, S., Tu, C., et al. (2017). Enzymatic hydrophilization of polyester fabrics using a recombinant cutinase Cut190 and their surface characterization. J. Fiber Sci. Technol. 73, 8-18. doi: 10.2115/fiberst.fiberst.2017-0002
Kawai, F., Oda, M., Tamashiro, T., Waku, T., Tanaka, N., Yamamoto, M., et al. (2014). A novel Ca2+-activated, thermostabilized polyesterase capable of hydrolyzing polyethylene terephthalate from Saccharomonospora viridis AHK 190. Appl. Microbiol. Biotechnol. 98, 10053-10064. doi: 10.1007/s00253-014$5860-\mathrm{y}$

Kay, M. J., Morton, L. H. G., and Prince, E. L. (1991). Bacterial degradation of polyester polyurethane. Int. Biodeterior. Biodegrad. 27, 205-222. doi: 10.1016/ 0265-3036(91)90012-G

Kenny, S. T., Runic, J. N., Kaminsky, W., Woods, T., Babu, R. P., Keely, C. M., et al. (2008). Up-cycling of PET (polyethylene terephthalate) to the biodegradable plastic PHA (polyhydroxyalkanoate). Environ. Sci. Technol. 42, 7696-7701. doi: 10.1021/es801010e

Kenny, S. T., Runic, J. N., Kaminsky, W., Woods, T., Babu, R. P., and O'Connor, K. E. (2012). Development of a bioprocess to convert PET derived terephthalic acid and biodiesel derived glycerol to medium chain length polyhydroxyalkanoate. Appl. Microbiol. Biotechnol. 95, 623-633. doi: 10.1007/ s00253-012-4058-4

Kijchavengkul, T., and Auras, R. (2008). Compostability of polymers. Polym. Int. 57, 793-804. doi: 10.1002/pi.2420

Kim, H. R., Lee, H. M., Yu, H. C., Jeon, E., Lee, S., Li, J. J., et al. (2020). Biodegradation of polystyrene by Pseudomonas sp. isolated from the gut of superworms (Larvae of Zophobas atratus). Environ. Sci. Technol. 54, 6987-6996. doi: 10.1021/acs.est.0c01495

Kim, M., Hyun, S., and Kwon, J.-H. (2015). Estimation of the environmental load of high- and low-density polyethylene from South Korea using a mass balance approach. Arch. Environ. Contam. Toxicol. 69, 367-373. doi: 10.1007/s00244015-0192-1

Kleeberg, I., Hetz, C., Kroppenstedt, R. M., Müller, R.-J., and Deckwer, W.-D. (1998). Biodegradation of aliphatic-aromatic copolyesters by Thermomonospora fusca and other thermophilic compost isolates. Appl. Environ. Microbiol. 64, 1731-1735. doi: 10.1128/AEM.64.5.1731-1735.1998

Kleeberg, I., Welzel, K., Vandenheuvel, J., Müller, R.-J., and Deckwer, W.-D. (2005). Characterization of a new extracellular hydrolase from Thermobifida fusca degrading aliphatic-aromatic copolyesters. Biomacromolecules 6, 262-270. doi: 10.1021/bm049582t

Klrbas, Z., Keskin, N., and Güner, A. (1999). Biodegradation of polyvinylchloride (PVC) by white rot fungi. Bull. Environ. Contam. Toxicol. 63, 335-342. doi: $10.1007 / \mathrm{s} 001289900985$

Kollattukudy, P. E. (1981). Structure, biosynthesis, and biodegradation of cutin and suberin. Annu. Rev. Plant Physiol. 32, 539-567. doi: 10.1146/annurev.pp.32. 060181.002543

Kundungal, H., Gangarapu, M., Sarangapani, S., Patchaiyappan, A., and Devipriya, S. P. (2019). Efficient biodegradation of polyethylene (HDPE) waste by the plastic-eating lesser waxworm (Achroia grisella). Environ. Sci. Pollut. Res. Int. 26, 18509-18519. doi: 10.1007/s11356-019-05038-9

Kyaw, B. M., Champakalakshmi, R., Sakharkar, M. K., Lim, C. S., and Sakharkar, K. R. (2012). Biodegradation of low density polythene (LDPE) by Pseudomonas species. Indian J. Microbiol. 52, 411-419. doi: 10.1007/s12088-012-0250-6

Lee, C. W., and Chung, J. D. (2009). Synthesis and biodegradation behavior of poly(ethylene terephthalate) oligomers. Polymer Korea 33, 198-202.

Li, W.-J., Jayakody, L. N., Franden, M. A., Wehrmann, M., Daun, T., Hauer, B., et al. (2019). Laboratory evolution reveals the metabolic and regulatory basis of ethylene glycol metabolism by Pseudomonas putida KT2440. Environ. Microbiol. 21, 3669-3682. doi: 10.1111/1462-2920.14703

Liebminger, S., Eberl, A., Sousa, F., Heumann, S., Fischer-Colbrie, G., CavacoPaulo, A., et al. (2007). Hydrolysis of PET and bis-(benzoyloxyethyl) terephthalate with a new polyesterase from Penicillium citrinum. Biocatal. Biotransform. 25, 171-177. doi: 10.1080/10242420701379734

Liu, X., Yu, L., Xie, F., Petinakis, E., Sangwan, P., Shen, S., et al. (2013). New evidences of accelerating degradation of polyethylene by starch. J. Appl. Polym. Sci. 130, 2282-2287. doi: 10.1002/app.39421

Lou, Y., Ekaterina, P., Yang, S. S., Lu, B., Liu, B., Ren, N., et al. (2020). Biodegradation of polyethylene and polystyrene by greater wax moth larvae (Galleria mellonella L.) and the effect of co-diet supplementation on the core gut microbiome. Environ. Sci. Technol. 54, 2821-2831. doi: 10.1021/acs.est.9b0 7044

Marques-Calvo, M. S., Cerda-Cuellar, M., Kint, D. P. R., Bou, J. J., and MunozGuerra, S. (2006). Enzymatic and microbial biodegradability of poly(ethylene 
terephthalate) copolymers containing nitrated units. Polym. Degrad. Stab. 91, 663-671. doi: 10.1016/j.polymdegradstab.2005.05.014

Matthews, S., Belcher, J. D., Tee, K. L., Girvan, H. M., Mclean, K. J., Rigby, S. E., et al. (2017). Catalytic determinants of alkene production by the cytochrome P450 peroxygenase OleTJE. J. Biol. Chem. 292, 5128-5143. doi: 10.1074/jbc. M116.762336

Mattioda, G., and Christidis, Y. (2000). "Glyoxylic acid," in Ullmann's Encyclopedia of Industrial Chemistry, eds M. Bohnet, C. G. Brinker, and B. Cornils (Weinheim: Wiley-VCH Verlag GmbH \& Co KGaA), 89-92.

Mohan, A. J., Sekhar, V. C., Bhaskar, T., and Nampoothiri, K. M. (2016). Microbial assisted high impact polystyrene (HIP) degradation. Bioresour. Technol. 213, 204-207. doi: 10.1016/j.biortech.2016.03.021

Mohanan, N., Sharma, P. K., and Levin, D. B. (2020). Characterization of an intracellular poly(3-hydroxyalkanoate) depolymerase from the soil bacterium, Pseudomonas putida LS46. Polym. Degrad. Stab. 175:109127. doi: 10.1016/j. polymdegradstab.2020.109127

Montazer, Z., Habibi Najafi, M. B., and Levin, D. B. (2019). Microbial degradation of low-density polyethylene and synthesis of polyhydroxyalkanoate polymers. Can. J. Microbiol. 65, 1-11. doi: 10.1139/cjm-2018-0335

Montazer, Z., Habibi Najafi, M. B., and Levin, D. B. (2020a). Challenges with verifying microbial degradation of polyethylene. Polymers 12:123. doi: 10.3390/ polym 12010123

Montazer, Z., Habibi Najafi, M. B., and Levin, D. B. (2020b). In vitro degradation of low-density polyethylene by new bacteria from larvae of the Greater Wax Moth, Galleria melonella. Can. J. Microbiol.

Mor, R., and Sivan, A. (2008). Biofilm formation and partial biodegradation of polystyrene by the actinomycete Rhodococcus ruber: biodegradation of polystyrene. Biodegradation 19, 851-858. doi: 10.1007/s10532-008-9188-0

Morancho, J. M., Ramis, X., Fernandez, X., Cadenato, A., Salla, J. M., et al. (2006). Calorimetric and thermogravimetric studies of UV irradiated polypropylene/starch-based materials aged in soil. Polym. Degrad. Stab. 91, 44-51. doi: 10.1016/j.polymdegradstab.2005.04.029

Motta, O., Proto, A., De Carlo, F., De Caro, F., Santoro, E., Brunetti, L., et al. (2009). Utilization of chemically oxidized polystyrene as co-substrate by filamentous fungi. Int. J. Hygiene Environ. Health 212, 61-66. doi: 10.1016/j.ijheh.2007. 09.014

Muckschel, B., Simon, O., Klebensberger, J., Graf, N., Rosche, B., Altenbuchner, J., et al. (2012). Ethylene glycol metabolism by Pseudomonas putida. Appl. Environ. Microbiol. 78, 8531-8539. doi: 10.1128/AEM.02062-12

Muller, R.-J., Kleeberg, I., and Deckwer, W.-D. (2001). Biodegradation of polyesters containing aromatic constituents. J. Biotechnol. 86, 87-95. doi: 10.1016/S01681656(00)00407-7

Müller, R.-J., Schrader, H., Profe, J., Dresler, K., and Deckwer, W.-D. (2005). Enzymatic degradation of poly(ethylene terephthalate): rapid hydrolysis using a hydrolase from T. fusca. Macromol. Rapid Commun. 26, 1400-1405. doi: 10.1002/marc. 200500410

Nakajima-Kambe, T., Onuma, F., Kimpara, N., and Nakahara, T. (1995). Isolation and characterization of a bacterium which utilizes polyester polyurethane as a sole carbon and nitrogen source. FEMS Microbiol. Lett. 129, 39-42. doi: 10.1111/j.1574-6968.1995.tb07554.x

Nakajima-Kambe, T., Shigeno-Akutsu, Y., Nomura, N., Onuma, F., and Nakahara, T. (1999). Microbial degradation of polyurethane, polyester polyurethanes and polyether polyurethanes. Appl. Microbiol. Biotechnol. 51, 134-140. doi: 10.1007/ s002530051373

Nakamiya, K., Sakasita, G., Ooi, T., and Kinoshita, S. (1997). Enzymatic degradation of polystyrene by hydroquinone peroxidase of Azotobacter beijerinckii HM121. J. Biosci. Bioeng. 84, 480-482. doi: 10.1016/S0922-338X(97) 82013-2

Nechwatal, A., Blokesch, A., Nicolai, M., Krieg, M., Kolbe, A., Wolf, M., et al. (2006). A contribution to the investigation of enzyme catalysed hydrolysis of poly(ethylene terephthalate) oligomers. Macromol. Mater. Eng. 291, 1486-1494. doi: 10.1002/mame.200600204

Neufeld, L., Stassen, F., Sheppard, R., and Gilman, T. (2016). The New Plastics Economy: Rethinking the Future of Plastics. Cologny: World Economic Forum.

Nikolic, V., Sava, V., Dusan, A., and Aleksander, P. (2013). Biodegradation of starch-graft-polystyrene and starch-graft-poly(methacrylic acid) copolymers in model river water. J. Serb. Chem. Soc. 78, 1425-1441. doi: 10.2298/ JSC121216051N
Nowak, B., Pają, K. J., Drozd-Bratkowicz, M., and Rymarz, G. (2011). Microorganisms participating in the biodegradation of modified polyethylene films in different soils under laboratory conditions. Int. Biodeterior. Biodegrad. 65, 757-767. doi: 10.1016/j.ibiod.2011.04.007

Oda, M., Yamagami, Y., Inaba, S., Oida, T., Yamamoto, M., Kitajima, S., et al. (2018). Enzymatic hydrolysis of PET: functional roles of three Ca2+ ions bound to a cutinase-like enzyme, Cut190*, and its engineering for improved activity. Appl. Microbiol. Biotechnol. 102, 10067-10077. doi: 10.1007/s00253-0189374-x

Odusanya, S. A., Nkwogu, J. V., Alu, N., Etuk Udo, G. A., Ajao, J. A., Osinkolu, G. A., et al. (2013). Preliminary studies on microbial degradation of plastics used in packaging potable water in Nigeria. Niger. Food J. 31, 63-72. doi: 10.1016/S0189-7241(15)30078-3

Ojeda, T. (2013). "Polymers and the environment," in Polymer Science, ed. F. Y $l$ lmaz (Rijeka: InTech), 1-34. doi: 10.5772/51057

Ojeda, T., Freitas, A., Birck, K., Dalmolin, E., Jacques, R., Bento, F., et al. (2011). Degradability of linear polyolefins under natural weathering. Polym. Degrad. Stab. 96, 703-707. doi: 10.1016/j.polymdegradstab.2010.12.004

Ojeda, T., Freitas, A., Dalmolin, E., Pizzol, M. D., Vignol, L., Melnik, J., et al. (2009). Abiotic and biotic degradation of oxo-biodegradable foamed polystyrene. Polym. Degrad. Stab. 94, 2128-2133. doi: 10.1016/j.polymdegradstab.2009.09. 012

O’Neill, A., Araújo, R., Casal, M., Guebitz, G., and Cavaco-Paulo, A. (2007). Effect of the agitation on the adsorption and hydrolytic efficiency of cutinases on polyethylene terephthalate fibres. Enzym. Microb. Technol. 40, 1801-1805. doi: 10.1016/j.enzmictec.2007.02.012

Osaki, T., Omotezako, M., Nagayama, R., Hirata, M., Iwanaga, S., Kasahara, J., et al. (1999). Horseshoe crab hemocyte-derived antimicrobial polypeptides, tachystatins, with sequence similarity to spider neurotoxins. J. Biol. Chem. 274, 26172-26178. doi: 10.1074/jbc.274.37.26172

Otake, Y., Kobayashi, T., Asabe, H., Murakami, N., and Ono, K. (1995). Biodegradation of low density polyethylene, polystyrene, polyvinyl chloride, and urea formaldehyde resin buried under soil for over 32 years. J. Appl. Polym. Sci. 56, 1789-1796. doi: 10.1002/app.1995.070561309

Owen, E. D. (1976). Photodegradation of polyvinyl chloride. ACS Symp. Ser. 25, 208-219. doi: 10.1021/bk-1976-0025.ch015

Owen, J. (2012). Degradation and Stabilisation of PVC. New York, NY: Springer Science \& Business Media.

Owen, S., Otani, T., Masaoka, S., and Ohe, T. (1996). The biodegradation of lowmolecular weight urethane compounds by a strain of Exophiala jeanselmei. Biosci. Biotechnol. Biochem. 60, 244-248. doi: 10.1271/bbb.60.244

Peixoto, J., Silva, L. P., and Krüger, R. H. (2017). Brazilian Cerrado soil reveals an untapped microbial potential for unpretreated polyethylene biodegradation. J. Hazard. Mater. 324, 634-644. doi: 10.1016/j.jhazmat.2016.11.037

Peng, B. Y., Chen, Z., Chen, J., Yu, H., Zhou, X., Criddle, C. S., et al. (2020a). Biodegradation of polyvinyl chloride (PVC) in Tenebrio molitor (Coleoptera Tenebrionidae) larvae. Environ. Int. 145:106106. doi: 10.1016/j.envint.2020. 106106

Peng, B. Y., Li, Y., Fan, R., Chen, Z., Chen, J., Brandon, A. M., et al. (2020b). Biodegradation of low-density polyethylene and polystyrene in superworms, larvae of Zophobas atratus (Coleoptera: Tenebrionidae): broad and limited extent depolymerization. Environ. Poll. 266:115206. doi: 10.1016/j.envpol.2020. 115206

Peng, B. Y., Su, Y., Chen, Z., Chen, J., Zhou, X., Benbow, M. E., et al. (2019). Biodegradation of polystyrene by dark (Tenebrio obscurus) and yellow (Tenebrio molitor) mealworms (Coleoptera: Tenebrionidae). Environ. Sci. technol. 53, 5256-5265. doi: 10.1021/acs.est.8b06963

Pirt, S. J. (1980). Microbial degradation of synthetic polymers. J. Chem. Technol. Biotechnol. 30, 176-179. doi: 10.1002/jctb.503300122

Plastics Additives (1998). Plastics Additives, ed. G. Pritchard (Dordrecht: Springer). Plastics Europe. (2017). Plastics - The Facts 2017: An Analysis of European Plastics Production, Demand and Waste Data 2017. Brussels: Plastics Europe.

Purdy, R. E., and Kolattukudy, P. E. (1975). Hydrolysis of plant cuticle by plant pathogens. Properties of cutinase I, cutinase II, and a nonspecific esterase isolated from Fusarium solani pisi. Biochemistry 14, 2832-2840. doi: 10.1021/ bi00684a007

Pushpadass, H. A., Weber, R. W., Dumais, J. J., and Hanna, M. A. (2010). Biodegradation characteristics of starch-polystyrene loose-fill foams in a 
composting medium. Bioresour. Technol. 101, 7258-7264. doi: 10.1016/j. biortech.2010.04.039

Ragaert, K., Delva, L., and Van Geem, K. (2017). Mechanical and chemical recycling of solid plastic waste. Waste Manage. 69, 24-58. doi: 10.1016/j.wasman.2017.07. 044

Rajandas, H., Parimannan, S., Sathasivam, K., Ravichandran, M., and Yin, L. S. (2012). A novel FTIR-ATR spectroscopy based technique for the estimation of low-density polyethylene biodegradation. Polym. Test. 31, 1094-1099. doi: 10.1016/j.polymertesting

Ramis, X., Cadenato, A., Salla, J. M., Morancho, J. M., Valles, A., Contat, L., et al. (2004). Thermal degradation of polypropylene/starch based materials with enhanced biodegradability. Polym. Degrad. Stab. 86, 483-491. doi: 10.1016/j. polymdegradstab.2004.05.021

Ren, L., Men, L., Zhang, Z., Guan, F., Tian, J., Wang, et al. (2019). Biodegradation of polyethylene by Enterobacter sp. D1 from the guts of Wax Moth Galleria mellonella. Int. J. Environ. Res. Publ. Health 16:1941. doi: 10.3390/ ijerph16111941

Restrepo-Flórez, J.-M., Bassi, A., and Thompson, M. R. (2014). Microbial degradation and deterioration of polyethylene. A review. Int. Biodeterior. Biodegrad. 88, 83-90. doi: 10.1016/j.ibiod.2013.12.014

Ribitsch, D., Herrero Acero, E., Greimel, K., Dellacher, A., Zitzenbacher, S., Marold, A., et al. (2012a). A new esterase from Thermobifida halotolerans hydrolyses polyethylene terephthalate (PET) and polylactic acid (PLA). Polymers 4, 617629. doi: 10.3390/polym 4010617

Ribitsch, D., Herrero Acero, E., Greimel, K., Eiteljoerg, I., Trotscha, E., Freddi, G., et al. (2012b). Characterization of a new cutinase from Thermobifida alba for PET-surface hydrolysis. Biocatal. Biotransform. 30, 2-9. doi: 10.3109/10242422. 2012.644435

Ribitsch, D., Herrero Acero, E., Przylucka, A., Zitzenbacher, S., Marold, A., Schwab, H., et al. (2015). Enhanced cutinase-catalyzed hydrolysis of polyethylene terephthalate by covalent fusion to hydrophobins. Appl. Environ. Microbiol. 81, 3586-3592. doi: 10.1128/AEM.04111-14

Ribitsch, D., Heumann, S., Trotscha, E., Acero, E. H., Greimel, K., Leber, R., et al. (2011). Hydrolysis of polyethyleneterephthalate by $p$-nitrobenzylesterase from Bacillus subtilis. Biotechnol. Progress 27, 951-960. doi: 10.1002/btpr.610

Ribitsch, D., Yebra, A. O., Zitzenbacher, S., Wu, J., Nowitsch, S., Steinkellner, G., et al. (2013). Fusion of binding domains to Thermobifida cellulosilytica cutinase to tune sorption characteristics and enhancing PET hydrolysis. Biomacromolecules 14, 1769-1776. doi: 10.1021/bm400140u

Riudavets, J., Salas, I., and Pons, M. J. (2007). Damage characteristics produced by insect pests in packaging film. J. Stored Prod. Res. 43, 564-570. doi: 10.1016/j. jspr.2007.03.006

Rojo, F. (2009). Degradation of alkanes by bacteria. Environ. Microbiol. 11, 24772490. doi: $10.1111 /$ j.1462-2920.2009.01948.x

Ronqvist, ÅM., Xie, W., Lu, W., and Gross, R. A. (2009). Cutinase-catalyzed hydrolysis of poly(ethylene terephthalate). Macromolecules 42, 5128-5138. doi: $10.1021 / \mathrm{ma} 9005318$

Roth, C., Wei, R., Oeser, T., Then, J., Foellner, C., Zimmermann, W., et al. (2014). Structural and functional studies on a thermostable polyethylene terephthalate degrading hydrolase from Thermobifida fusca. Appl. Microbiol. Biotechnol. 98, 7815-7823. doi: 10.1007/s00253-014-5672-0

Rowe, L., and Howard, G. T. (2002). Growth of Bacillus subtilis on polyurethane and the purification and characterization of a polyurethanase-lipase enzyme. Int. Biodeterior. Biodegrad. 50, 33-40. doi: 10.1016/S0964-8305(02)00047-1

Rübsam, K., Davari, M. D., Jakob, F., and Schwaneberg, U. (2018a). KnowVolution of the polymer-binding peptide LCI for improved polypropylene binding. Polymers 10:423. doi: 10.3390/polym10040423

Rübsam, K., Weber, L., Jakob, F., and Schwaneberg, U. (2018b). Directed evolution of polypropylene and polystyrene binding peptides. Biotechnol. Bioeng. 115, 321-330. doi: 10.1002/bit.26481

Ruiz, C., Main, T., Hilliard, N. P., and Howard, G. T. (1999). Purification and characterization of twopolyurethanase enzymes from Pseudomonas chlororaphis. Int. Biodeterior. Biodegrad. 43, 43-47. S0964-8305(98)00067-5 doi: 10.1016/S0964-8305(98)00067-5

Sahebnazar, Z., Shojaosadati, S. A., Mohammad-Taheri, M., and Nosrati, M. (2010). Biodegradation of low-density polyethylene (LDPE) by isolated fungi in solid waste medium. Waste Manage. 30, 396-401. doi: 10.1016/j.wasman.2009.09. 027

Sajtos, A. (1991). Process for the Preparation of Glyoxylic Acid and Glyoxylic Acid Derivates. US Patent No 5,015,760.
Sameh, A. S., Alariqi, Pradeep Kumar, A., Rao, B. S. M., and Singh, R. P. (2006). Biodegradation of $\gamma$-sterilized biomedical polyolefins under composting and fungal culture environments. Polym. Degrad. Stab. 91, 1105-1116. doi: 10.1016/ j.polymdegradstab.2005.07.004

Santo, M., Weitsman, R., and Sivan, A. (2013). The role of the copper-binding enzyme, laccase, in the biodegradation of polyethylene by the actinomycete Rhodococcus ruber. Int. Biodeterior. Biodegrad. 84, 204-210. doi: 10.1016/j. ibiod.2012.03.001

Schlemmer, D., Sales, M. J. A., and Resck, I. S. (2009). Degradation of different polystyrene/thermoplastic starch blends buried in soil. Carb. Polym. 75, 58-62. doi: 10.1016/j.carbpol.2008.06.010

Sekhar, V. C., Nampoothiri, K. M., Mohan, A. J., Nair, N. R., Bhaskar, T., and Pandey, A. (2016). Microbial degradation of high impact polystyrene (HIPS), an e-plastic with decabromodiphenyl oxide and antimony trioxide. J. Hazard. Mater. 318, 347-354. doi: 10.1016/j.jhazmat.2016.07.008

Sen, S. K., and Raut, S. (2016). Microbial degradation of low density polyethylene (LDPE): a review. J. Environ. Chem. Eng. 3, 462-473. doi: 10.1016/j.jece.2015. 01.003

Seppala, J., Linko, Y. Y., and Su, T. (1991). Photo and biodegradation of high volume thermoplastics, Acta polytechnica scandinavica. J. Chem. Technol. Metall. 198:33.

Shah, A. A., Hasan, F., Hameed, A., and Ahmed, S. (2008). Biological degradation of plastics: a comprehensive review. Biotechnol. Adv. 26, 246-265. doi: 10.1016/ j.biotechadv.2007.12.005

Shah, M. M., Barr, D. P., Chung, N., and Aust, S. D. (1992). Use of white rot fungi in the degradation of environmental chemicals. Toxicol. Lett. 64, 493-501. doi: 10.1016/0378-4274(92)90224-8

Shang, J., Chai, M., and Zhu, Y. (2003). Photocatalytic degradation of polystyrene plastic under fluorescent light. Environ. Sci. Technol. 37, 4494-4499. doi: 10. 1021/es0209464

Sharma, S., and Chatterjee, S. (2017). Microplastic pollution, a threat to marine ecosystem and human health: a short review. Environ. Sci. Pollut. Res. 24, 21530-21547. doi: 10.1007/s11356-017-9910-8

Shimpi, N., Mishra, S., and Kadam, M. (2012). Biodegradation of polystyrene (PS)-poly(lactic acid) (PLA) nanocomposites using Pseudomonas aeruginosa. Macromol. Res. 20, 181-187. doi: 10.1007/s13233-012-0026-1

Sielicki, M., Focht, D. D., and Martin, J. P. (1978). Microbial degradation of (C14C) polystyrene and 1,3-diphenylbutane. Can. J. Microbiol. 24, 798-803. doi: $10.1139 / \mathrm{m} 78-134$

Silva, C., Da, S., Silva, N., Matama, T., Araujo, R., Martins, M., et al. (2011). Engineered Thermobifida fusca cutinase with increased activity on polyester substrates. Biotechnol. J. 6, 1230-1239. doi: 10.1002/biot.201000391

Song, Y., Qiu, R., Hu, J., Li, X., Zhang, X., Chen, Y., et al. (2020). Biodegradation and disintegration of expanded polystyrene by land snails Achatina fulica. Sci. Total Environ. 746:141289. doi: 10.1016/j.scitotenv.2020.141289

Stern, R. V., and Howard, G. T. (2000). The polyester polyurethanase gene (pueA) from Pseudomonas chlororaphis encodes a lipase. FEMS Microbiol. Lett. 185, 163-168. doi: 10.1111/j.1574-6968.2000.tb09056.x

Stinson, S. (1987). Discoverers of polypropylene share prize. Chem. Eng. News 65:30. doi: 10.1021/cen-v065n010.p030

Sudhakar, M., Doble, M., Sriyutha Murthy, P., and Venkatesan, R. (2008). Marine microbe-mediated biodegradation of low- and high-density polyethylenes. Int. Biodeterior. Biodegrad. 61, 203-213. doi: 10.1016/j.ibiod.2007.07.011

Syranidou, E., Karkanorachaki, K., Amorotti, F., Repouskou, E., Kroll, K., Kolvenbach, B., et al. (2017). Development of tailored indigenous marine consortia for the degradation of naturally weathered polyethylene films. PLoS One 12:e0183984. doi: 10.1371/journal.pone.0183984

Takei, D., Washio, K., and Morikawa, M. (2008). Identification of alkane hydroxylase genes in Rhodococcus sp. strain TMP2 that degrades a branched alkane. Biotechnol. Lett. 30, 1447-1452. doi: 10.1007/s10529-008-9710-9

Tokiwa, Y., and Calabia, B. P. (2007). Biodegradability and biodegradation of polyesters. J. Polym. Environ. 15, 259-267. doi: 10.1007/s10924-007-0066-3

Tokiwa, Y., Calabia, B. P., Ugwu, C. U., and Aiba, S. (2009). Biodegradability of plastics. Int. J. Mol. Sci. 10, 3722-3742. doi: 10.3390/ijms10093722

Torikai, A., and Hasegawa, H. (1999). Accelerated photodegradation of poly (vinyl chloride). Polym. Degrad. Stab. 63, 441-445. doi: 10.1016/S0141-3910(98) 00125-6

Usha, R., Sangeetha, T., and Palaniswamy, M. (2011). Screening of polyethylene degrading microorganisms from garbage soil. Libyan Agric. Res. Center J. Int. 2, 200-204. 
Vega, R. E., Main, T., and Howard, G. T. (1999). Cloning and expression in Escherichia coli of apolyurethane-degrading enzyme from Pseudomonas fluorescens. Int. Biodeterior. Biodegrad. 43, 49-55. doi: 10.1016/S0964-8305(98) 00068-7

Venkatachalam, S., Nayak, S. G., Labde, J. V., Gharal, P. R., Rao, K., Kelkar, A. K. (2012). "Degradation and Recyclability of poly (ethylene terephthalate)," in Polyester, ed. H. E.-D. Saleh, London: InTech. doi: 10.5772/48612

Verce, M. F., Ulrich, R. L., and Freedman, D. L. (2000). Characterization of an isolate that uses vinyl chloride as a growth substrate under aerobic conditions. Appl. Environ. Microbiol. 66, 3535-3542. doi: 10.1128/AEM.66.8.3535-3542. 2000

Vertommen, M. A. M. E., Nierstrasz, V. A., van der Veer, M., and Warmoeskerken, M. M. C. G. (2005). Enzymatic surface modification of poly(ethylene terephthalate). J. Biotechnol. 120, 376-386. doi: 10.1016/j.jbiotec.2005.06.015

Wallace, P. W., Haernvall, K., Ribitsch, D., Zitzenbacher, S., Schittmayer, M., Steinkellner, G., et al. (2017). PpEst is a novel PBAT degrading polyesterase identified by proteomic screening of Pseudomonas pseudoalcaligenes. Appl. Microbiol. Biotechnol. 101, 2291-2303. doi: 10.1007/s00253-016-7992-8

Ward, P. G., Goff, M., Donner, M., Kaminsky, W., and O'Connor, K. E. (2006). A two-step chemo-biotechnological conversion of polystyrene to a biodegradable thermoplastic. Environ. Sci. Technol. 40, 2433-2437. doi: 10.1021/es0517668

Watanabe, M., Kawai, F., Shibata, M., Yokoyama, S., and Sudate, Y. (2003). Computational method for analysis of polyethylene biodegradation. J. Comput. Appl. Math. 161, 133-144. doi: 10.1016/S0377-0427(03)00551-X

Webb, H. K., Arnott, J., Crawford, R. J., and Ivanova, E. P. (2013). Plastic degradation and its environmental implications with special reference to poly(ethylene terephthalate). Polymers 5, 1-18. doi: 10.3390/polym5010001

Wehrmann, M., Billard, P., Martin-Meriadec, A., Zegeye, A., and Klebensberger, J. (2017). Functional role of lanthanides in enzymatic activity and transcriptional regulation of pyrroloquinoline quinone-dependent alcohol dehydrogenases in Pseudomonas putida KT2440. mBio 8:e00570-17. doi: 10.1128/mBio.00570- 17

Wei, R., Oeser, T., Barth, M., Weigl, N., Lübs, A., Schulz-Siegmund, M., et al. (2014a). Turbidimetric analysis of the enzymatic hydrolysis of polyethylene terephthalate nanoparticles. J. Mol. Catal. BEnzym. 103, 72-78. doi: 10.1016/ j.molcatb.2013.08.010

Wei, R., Oeser, T., Then, J., Kühn, N., Barth, M., and Zimmermann, W. (2014b). Functional characterization and structural modeling of synthetic polyesterdegrading hydrolases form Thermomonospora curvata. AMB Express 4:44. doi: 10.1186/s13568-014-0044-9

Wei, R., and Zimmermann, W. (2017). Microbial enzymes for the recycling of recalcitrant petroleum-based plastics: how far are we? Microb. Biotechnol. 10, 1308. doi: 10.1111/1751-7915.12710

Weiland, M., Daro, A., and David, C. (1995). Biodegradation of thermally oxidised polyethylene. Polym. Degrad. Stab. 48, 275-289. doi: 10.1016/0141-3910(95) 00040-S

Welzel, K., Müller, R. J., and Deckwer, W. D. (2002). Enzymatischer Abbau von polyester-nanopartikeln. Chem. Ingenieur Technik 74, 1496-1500. doi: 10.1002/ 1522-2640(20021015)74:10<1496::AID-CITE1496>3.0.CO;2-P

Wilkes, R. A., and Aristilde, L. (2017). Degradation and metabolism of synthetic plastics and associated products by Pseudomonas sp.: capabilities and challenges. J. Appl. Microbiol. 123, 582-593. doi: 10.1111/jam.13472

Wool, R. P., Raghavan, D., Wagner, G. C., and Billieux, S. (2000). Biodegradation dynamics of polymer-starch composites. J. Appl. Polym. Sci. 77, 1643-1657. doi: 10.1002/1097-4628(20000822)77:8<1643::AID-APP1>3.0.CO;2-8

Xu, J., Cui, Z., Nie, K., Cao, H., Jiang, M., Xu, H., et al. (2019). A quantum mechanism study of the $\mathrm{C}$-C bond cleavage to predict the bio-catalytic polyethylene degradation. Front. Microbiol. 10:489. doi: 10.3389/fmicb.2019. 00489

Yang, J., Yang, Y., Wu, W. M., Zhao, J., and Jiang, L. (2014). Evidence of polyethylene biodegradation by bacterial strains from the guts of plastic-eating waxworms. Environ. Sci. Technol. 48, 13776-13784. doi: 10.1021/es504038a

Yang, L., Gao, J., Liu, Y., Zhuang, G., Peng, X., Wu, W.-M., et al. (2021). Biodegradation of expanded polystyrene and low-density polyethylene foams in larvae of Tenebrio molitor Linnaeus (Coleoptera: Tenebrionidae): broad versus limited extent depolymerization and microbe-dependence versus independence. Chemosphere 262:127818. doi: 10.1016/j.chemosphere.2020. 127818
Yang, S.-S., Brandon, A. M., Andrew Flanagan, J. C., Yang, J., Ning, D., Cai, S.Y. Y., et al. (2018a). Biodegradation of polystyrene wastes in yellow mealworms (larvae of Tenebrio molitor Linnaeus): factors affecting biodegradation rates and the ability of polystyrene-fed larvae to complete their life cycle. Chemosphere 191, 979-989. doi: 10.1016/j.chemosphere.2017.10.117

Yang, S.-S., Wu, W. M., Brandon, A. M., Fan, H. Q., Receveur, J. P., Li, Y., et al. (2018b). Ubiquity of polystyrene digestion and biodegradation within yellow mealworms, larvae of Tenebrio molitor Linnaeus (Coleoptera: Tenebrionidae). Chemosphere 212, 262-271.

Yang, Y., Chen, J., Wu, W.-M., Zhao, J., and Yang, J. (2015). Complete genome sequence of Bacillus sp.YP1, a polyethylene-degrading bacterium from waxworm's gut. J. Biotechnol. 200, 77-78. doi: 10.1016/j.jbiotec.2015.02.034

Yang, Y., Yang, J., Wu, W. M., Zhao, J., Song, Y., Gao, L., et al. (2015a). Biodegradation and mineralization of polystyrene by plastic-eating mealworms. 1. Chemical and physical characterization and isotopic tests. Environ. Sci. Technol. 49:12080. doi: 10.1021/acs.est.5b02661

Yang, Y., Yang, J., Wu, W. M., Zhao, J., Song, Y., Gao, L., et al. (2015b). Biodegradation and mineralization of polystyrene by plastic-eating mealworms: Part 2. Role of gut microorganisms. Environ. Sci. Technol. 49, 12087-12093. doi: 10.1021/acs.est.5b02663

Yin, C.-F., Xu, Y., and Zhou, N.-Y. (2020). Biodegradation of polyethylene mulching films by a co-culture of Acinetobacter sp. strain NyZ450 and Bacillus sp. strain NyZ451 isolated from Tenebrio molitor larvae. Int. Biodeterior. Biodegrad. 155:105089. doi: 10.1016/j.ibiod.2020.105089

Yoon, M. G., Jeon, J. H., and Kim, M. N. (2012). Biodegradation of polyethylene by a soil bacterium and AlkB cloned recombinant cell. J. Bioremed. Biodegrad. 3:145.

Yoshida, S., Hiraga, K., Takehara, T., and Oda, K. (2016). A bacterium that degrades and assimilates poly(ethylene terephthalate). Science 351, 1196-1199. doi: 10. 1126/science.aad6359

Yue, H., Zhao, Y., Ma, X., and Gong, J. (2012). Ethylene glycol. Properties, synthesis, and applications. Chem. Soc. Rev. 41, 4218-4244. doi: 10.1039/ c2cs15359a

Zhang, Y., Chen, S., Xu, M., Cavaco-Paulo, A., Wu, J., and Chen, J. (2010) Characterization of Thermobifida fusca cutinase-carbohydrate-binding module fusion proteins and their potential application in bioscouring. Appl. Environ. Microbiol. 76, 6870-6876. doi: 10.1128/AEM.00896-10

Zheng, Y., and Yanful, E. K. (2005). A review of plastic waste degradation. Crit. Rev. Biotechnol. 25, 243-250.

Zheng, Y., Yanful, E. K., and Bassi, A. S. (2005). A review of plastic waste biodegradation. Crit. Rev. Biotechnol. 25, 243-250. doi: 10.1080/ 07388550500346359

Zimmermann, W., and Billig, S. (2011). Enzymes for the biofunctionalization of poly(ethylene terephthalate). Adv. Biochem. Engin/Biotechnol. 125, 97-120. doi: 10.1007/10_2010_87

Zuchowska, D., Hlavata, D., Steller, R., Adamiak, W., and Meissner, W. (1999). Physical structure of polyolefin-starch blends after ageing. Polym. Degrad. Stab. 64, 339-347. doi: 10.1016/S0141-3910(98)00212-2

Zuchowska, D., Steller, R., and Meissner, W. (1998). Structure and properties of degradable polyolefin-starch blends. Polym. Degrad. Stab. 60, 471-480. doi: 10.1016/S0141-3910(97)00110-9

Zumstein, M. T., Rechsteiner, D., Roduner, N., Perz, V., Ribitsch, D., Guebitz, G. M., et al. (2017). Enzymatic hydrolysis of polyester thin films at the nanoscale: effects of polyester structure and enzyme active-site accessibility. Environ. Sci. Technol. 51, 7476-7485. doi: 10.1021/acs.est.7b01330

Conflict of Interest: The authors declare that the research was conducted in the absence of any commercial or financial relationships that could be construed as a potential conflict of interest.

Copyright (C) 2020 Mohanan, Montazer, Sharma and Levin. This is an open-access article distributed under the terms of the Creative Commons Attribution License (CC BY). The use, distribution or reproduction in other forums is permitted, provided the original author(s) and the copyright owner(s) are credited and that the original publication in this journal is cited, in accordance with accepted academic practice. No use, distribution or reproduction is permitted which does not comply with these terms. 